\title{
Mathematical modelling and numerical appproxi- mation of a leachate flow in the anaerobic biodegra- dation of waste in a landfill
}

\author{
Z. Belhachmi ${ }^{1}$, Z. Mghazli ${ }^{2}$ and S. Ouchtout ${ }^{1,2, *}$ \\ ${ }^{1}$ University of Haute-Alsace, IRIMAS UR 7499, F-68100 Mulhouse, France, Uni- \\ versity of Strasbourg, France \\ ${ }^{2}$ Interdisciplinary Laboratory for Natural Resources and Environment, Ibn Tofaï \\ University, Kénitra, Morocco
}

\begin{abstract}
We consider a coupled PDE-ODEs model that describes the bacterial dynamics of the anaerobic biodegradation of waste in a landfill taken as an unsatureted porous medium. We add a diffusion term in the ODEs part which gives a rise to a reactiondiffusion system coupled with porous media equations. This yields a new model of the biodegradation of the waste where the biological variables are varying in space and time. We perform the mathematical analysis of the resulting system and we consider a discretization based on BDF schemes and $P_{1}$ conforming finite elements for the reactiondiffusion subsystem and a mixed finite elements $R T_{0} / P_{0}$ for the Darcy flow. We carry out the numerical analysis and we obtain the expected a priori estimates. We perform several numerical 2D and 3D simulations which are in agreement with the theoretical results.
\end{abstract}

AMS subject classifications: 65M10, 78A48

Key words: Anaerobic biodegradation, Leachate flow, Biogas production, Coupled problem, Mixed finite element.

\section{Introduction}

Waste management and renewable energy generation are two key issues in nowadays societies. A major research field arising in recent years focuses on combining the two aforementioned topics by developing new techniques to handle waste and to use it in the energy production. The anaerobic digestion process is a natural biological process of decomposition of organic mater by microorganisms (bacteria) that are activated under anaerobic conditions, that is to say without oxygen. It is characterized by a succession of complex reactions both in parallel and in series. In the long term, the organic mater is transformed into biogas, a mixture mainly composed of methane and carbon dioxide. The main stages of this process are hydrolysis, acidogenesis, acetogenesis and methanogenesis. Models such

\footnotetext{
${ }^{*}$ Corresponding author. Email addresses: zakaria.belhachmi@uha.fr (Z. Belhachmi), zoubida.mghazli@uit.ac.ma (Z. Mghazli), salih.ouchtout@uha.fr (S. Ouchtout)
} 
as the "ADM1" allow a detailed description of this process, cf. [25]. However, such complex models are not well suited for mathematical analysis. A large number of simpler models have been investigated in the literature over theses last years $[15,17,18,33,34,36]$. When dealing with the digestion of wastewater, it is recognized that the limiting step is the methanoganesis. In such a case, modelling include one-, two- or three-step models. Of particular interest is the model by Bernard et al., 2001 ( [7]) which proposes to model the anaerobic digestion process as a two-step process involving both the acidogenesis (using a Monod kinetics) and the methanogenesis processes (using a Haldane kinetics). This model, for which the mathematical analysis has been conducted by Benyahia et al., 2012, cf. [6], is very popular, notably for control purposes, since it remains of moderate complexity while being quite easy to calibrate to predict process behaviour with satisfying performances, $[4,16]$ or still [27]. It has also been the basis for proposing a systematic way to link simple models to ADM1 predictions, cf. [25].

The waste landfill is a multiphase medium consisting of solid, liquid and gas phases and is considered as a porous medium. The biodegradation process produces the biogas and leachate in the landfill [32] and thus the coupling of the biological activity and the fluid flow. We refer the interested reader to the review paper of Agostini, Sundberg, Navia [1] on this subject.

In this article, we present a new coupled model combining the biological and the mechanical aspects describing respectively the process of the biogas production and the leachate flow during the anaerobic biodegradation of the organic mater in a landfill. We give, as in [31], the standard two-step approach of the biogas production with two different specific growth rates. We modify the model by transforming the ordinary differential equations part into partial differential equations (PDEs) to obtain a system of diffusion-reaction. The added diffusion allows us to better represent the bacterial dynamic in the spatial domain and to take into account the nonhomogeneous distribution of methanogenese bacteria which is a more realistic assumption in a waste collection sites ( [37], [30], [40]). Secondly, we give the full system governing the leachate flow and the biodegradation.

Besides the fact that describing a part of the bacterial dynamic as a diffusion-reaction system leads to more realistic and accurate modelling, it also acts as a regularization procedure for the initial ODEs system for which we have established in [31] the existence of several (stable and unstable) equilibria.

We consider discrete problems based on high order time discretization and conforming finite elements for the reaction-diffusion system and a mixed finite elements discretization (Raviart-Thomas $/ P_{0}$ ) for the porous medium equations. We perform their numerical analysis and we obtain optimal a priori error estimates. We present some 2D and 3D numerical results that show the relevance of the new model. This study constitutes an important step towards the more suitable model of the dynamic of the waste in a landfill with multiple phases (gaz/liquid/solid).

The rest of this article is organized as follows: In section 2. we derive the mathematical model governing the chemical and physical phenomena taking place in the waste collection site. In section 3. we establish the existence and uniqueness results for the system and we perform the semi-discretization in time based on the second order BDF2 scheme 
and we derive the variational formulation for the full problem. Section 4. is devoted to the numerical analysis of the PDEs system. We use different discrete setting between the reaction diffusion subsystem, for which we use $P_{1}$-conforming finite element approximation and the porous medium subsystem that we discretize as mixed variational formulation in the velocity-pressure variables, with Raviart-Thomas $R T_{0}$ for the velocity and $P_{0}$ finite elements for the pressure. We carry out the numerical analysis and we derive a priori error estimates in the spirits of [19] [5]. In section 5. we present some 2D and 3D numerical simulations and we detail the method for solving the proposed model.The implementation is based on the open source software FreeFem++ [26].

\section{A coupled mathematical model for the biological dynamic and the flow}

\subsection{The bacterial dynamic}

\subsubsection{The anaerobic digestion system in two-step}

The methane production process considered in this article is described as follows: a first step of hydrolysis / acidogenesis of the organic mater, represented by its concentration that we denote $X$, leads to the formation of carbon dioxide $\left(\mathrm{CO}_{2}\right)$ and simple soluble organic mater $S$. The latter is used as a substrate by methanogenic bacteria $B$ which in turn produce carbon dioxide $\left(\mathrm{CO}_{2}\right)$ as well as methane $\left(\mathrm{CH}_{4}\right)$. At their death, the methanogenic bacteria in turn constitute a complex substrate to hydrolysis step (see FIGURE (1)). The principle of

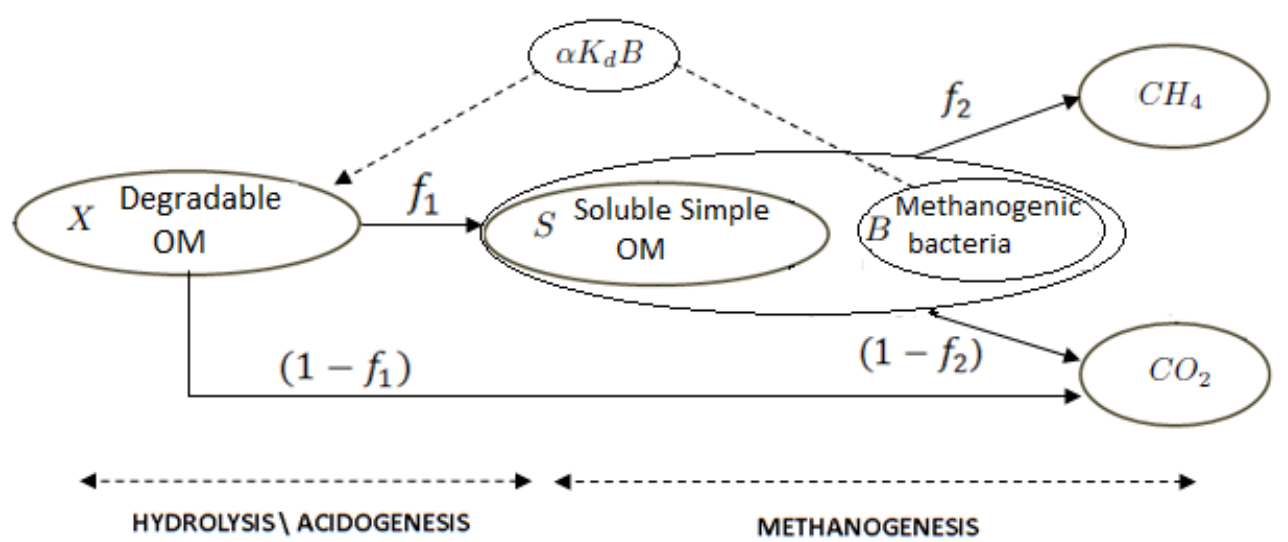

Figure 1: Scheme used for modelling anaerobic degradation of organic mater.

mass conservation and the law of bacterial growth, show that the bacterial dynamic and the 
biogas production are solutions of the following system of ordinary differential equations

$$
\left\{\begin{array}{l}
\frac{d X}{d t}=-K_{h} X+\alpha K_{d} B \\
\frac{d B}{d t}=\left(\mu(S)-K_{d}\right) B \\
\frac{d S}{d t}=f_{1} K_{h} X-\frac{1}{Y} \mu(S) B \\
\frac{d\left[C O_{2}\right]}{d t}=\left(1-f_{1}\right) K_{h} X+\left(1-f_{2}\right) \frac{1-Y}{Y} \mu(S) B \\
\frac{d\left[C H_{4}\right]}{d t}=f_{2} \frac{1-Y}{Y} \mu(S) B .
\end{array}\right.
$$

The parameters $f_{1}$ and $f_{2}$ are the stoichiometric coefficients which represent the parts of organic mater (OM) transformed into simple OM or methane during the "hydrolysis / acidogenesis" step or methanogenesis step respectively. Consequently $\left(1-f_{1}\right)$ and $\left(1-f_{2}\right)$ represent the parts of $\mathrm{OM}$ transformed into $\mathrm{CO}_{2}$ during these two steps respectively. We have denoted by $K_{h}$ the rate of hydrolysis, $Y$ the rate of use of the substrate, $K_{d}$ the bacterial mortality rate and $\alpha$ is a constant $0<\alpha \leq 1$ representing the fraction of the biomass mortality reused as a substrate in the methanogenesis. The dynamic variables of the system (2.1) are the degradable complex material $X$, the methanogenic biomass $B$, the soluble organic mater $S$, the carbon dioxide $\left[\mathrm{CO}_{2}\right]$ and the methane $\left[\mathrm{CH}_{4}\right]$. This model was proposed by Rouez in [36] with Monod law and $\alpha=1$. Several laws exist for the specific growth rate $\mu(S)$. The most used are the Monod law

$$
\mu(S)=\frac{\mu_{m} S}{K_{S}+S}
$$

and the Haldane law

$$
\mu(S)=\frac{\mu_{m} S}{K_{S}+S+\frac{S^{2}}{K_{I}}}
$$

where $\mu_{m}$ is the maximum growth rate, $K_{S}$ is the half-saturation constant and $K_{I}$ is the inhibition constant. The Monod law is related to saturation and limitation phenomena and the Haldane law is related to saturation and inhibition phenomena [7]. In this article we will compare the two laws. The system (2.1) allows us to compute the global rate of production of the biogas which constitutes the main part of the source/sink term in the PDEs model of the flow in the waste. The asymptotic behavior analysis of this ODEs system is performed in [31] and some coupled ODEs-PDEs system in this field are studied in [20] [13] [14] [21]. In this article, we consider the realistic case of nonhomogenous landfill where we have to take into account the spatial dependency of the bacterial dynamic (represented by the variables of the ODEs system). This leads us to transform the ODEs system into a PDE system of diffusion-reaction form. 


\subsubsection{The reaction-diffusion PDEs system}

Let $\Omega$ be an open bounded subset of $\mathbb{R}^{d}, d \geq 2$, representing the landfill. We suppose that its boundary $\Gamma:=\partial \Omega$ is Lipschitz-continuous and is the union of two parts $\Gamma_{D}$ and $\Gamma_{N}$ where Dirichlet and Neumann boundary conditions are respectively imposed, with $\Gamma_{D} \cap \Gamma_{N}=\emptyset$. The outward normal vector on $\Gamma$ will be noted $\mathbf{n}$. For $T>0$, the time interval is $[0, T]$. We write $\left.Q_{T}=\Omega \times\right] 0, T\left[\right.$ and $\left.\Gamma_{T}=\Gamma \times\right] 0, T[$. The following system is obtained by adding the diffusion terms into the system (2.1) which describe the molecular agitation of the dynamic quantities ( [30], [37], [40]).

$$
\begin{cases}\frac{d X}{d t}=-K_{h} X+\alpha K_{d} B+\operatorname{div}\left(D_{1} \nabla X\right) & \text { in } \quad \Omega \times] 0, T[ \\ \frac{d B}{d t}=\left(\mu(S)-K_{d}\right) B+\operatorname{div}\left(D_{2} \nabla B\right) & \text { in } \quad \Omega \times] 0, T[ \\ \frac{d S}{d t}=f_{1} K_{h} X-\frac{1}{Y} \mu(S) B+\operatorname{div}\left(D_{3} \nabla S\right) & \text { in } \quad \Omega \times] 0, T[\end{cases}
$$

where $D_{1}, D_{2}$ and $D_{3}$ are, respectively, the diffusion coefficients of $X, B$ and $S$. The equations on the concentrations $\mathrm{CO}_{2}$ and $\mathrm{CH}_{4}$ are not modified because their simple structure shows that they vary in space with the other variables. Therefore, to not increase the computational cost we do not add any diffusion to these equations a priori. Thus, the last equations in the system (2.4), remain unchanged. However, in the numerical section, we simulate and compare the different cases, including with added diffusion to the last variables, $\left(\mathrm{CO}_{2}\right)$ and $\left(\mathrm{CH}_{4}\right)$ (complete diffusion vs partial diffusion). Obviously, the analysis and the discretization remain essentially unchanged.We will denote by $\overline{\bar{D}}^{c}$ the complete diffusion, that means the coefficients diffusion for $\left(\mathrm{CO}_{2}\right)$ and $\left(\mathrm{CH}_{4}\right)$ are added.

BOUNDARY AND INITIAL CONDITIONS OF THE SYSTEM (2.4)

We fix a time interval of biogas production $[0, T]$ where $T$ corresponds to the moment when the leachate begins to stagnate at the bottom of the domain, or the moment when it is necessary to recover the biogas. We take the following no-flux boundary conditions associated with the system (2.4)

$$
\left.D_{1} \frac{\partial X}{\partial \mathbf{n}}=D_{2} \frac{\partial B}{\partial \mathbf{n}}=D_{3} \frac{\partial S}{\partial \mathbf{n}}=0 \text { on } \Gamma \times\right] 0, T[.
$$

The initial conditions are given as follows

$$
\begin{gathered}
X(0, x)=X_{0}(x), \quad B(0, x)=B_{0}(x), \quad S(0, x)=S_{0}(x) \quad \text { in } \Omega, \\
{\left[\mathrm{CO}_{2}\right](0, x)=\left[\mathrm{CO}_{2}\right]_{0}(x), \quad\left[\mathrm{CH}_{4}\right](0, x)=\left[\mathrm{CH}_{4}\right]_{0}(x) \text { in } \Omega .}
\end{gathered}
$$

We set

$$
\mathbf{U}=\left(u_{1}, u_{2}, u_{3}\right)^{t}=(X, B, S)^{t}, \quad \mathbf{G}=\left(u_{4}, u_{5}\right)^{t}=\left(\left[\mathrm{CO}_{2}\right],\left[C \mathrm{CH}_{4}\right]\right)^{t}
$$


and the diffusion coefficients matrix $\overline{\bar{D}}$ is defined as follows

$$
\overline{\bar{D}}=\left(\begin{array}{ccc}
D_{1} & 0 & 0 \\
0 & D_{2} & 0 \\
0 & 0 & D_{3}
\end{array}\right)
$$

The right-hand members of the equations in (2.1) will be denoted by the functions $F_{i}, i=$ $1,2, . ., 5$ defined as follows

$$
\begin{aligned}
& F_{1}(\mathbf{U})=-K_{h} u_{1}+\alpha K_{d} u_{2}, \quad F_{2}(\mathbf{U})=\left(\mu\left(u_{3}\right)-K_{d}\right) u_{2}, \\
& F_{3}(\mathbf{U})=f_{1} K_{h} u_{1}-\frac{1}{Y} \mu\left(u_{3}\right) u_{2}, \quad F_{5}(U)=f_{2} \frac{1-Y}{Y} \mu\left(u_{3}\right) u_{2}, \\
& F_{4}(\mathbf{U})=\left(1-f_{1}\right) K_{h} u_{1}+\left(1-f_{2}\right) \frac{1-Y}{Y} \mu\left(u_{3}\right) u_{2},
\end{aligned}
$$

and we set the vector functions $\mathbf{F}^{\mathbf{1}}$ and $\mathbf{F}^{2}$ such that

$$
\mathbf{F}^{1}(\mathbf{U})=\left(F_{1}(U), F_{2}(U), F_{3}(U)\right)^{T}, \quad \mathbf{F}^{2}(U)=\left(F_{4}(U), F_{5}(U)\right)^{T} .
$$

The system (2.4) can be written:

$$
\begin{cases}\frac{\partial \mathbf{U}}{\partial t}-\operatorname{div}(\overline{\bar{D}} \nabla \mathbf{U})=\mathbf{F}^{\mathbf{1}}(\mathbf{U}) & \text { in } \Omega \times] 0, T[ \\ \frac{d \mathbf{G}}{d t}=\mathbf{F}^{2}(\mathbf{U}) & \text { in } \Omega \times] 0, T[ \\ \overline{\bar{D}} \frac{\partial \mathbf{U}}{\partial n}=0 & \text { on } \partial \Omega \times] 0, T[ \\ \mathbf{U}(0, \cdot)=\mathbf{U}^{0}(\cdot), \quad \mathbf{G}(0, \cdot)=\mathbf{G}^{0}(\cdot) & \text { in } \Omega .\end{cases}
$$

Remark 2.1. From the $2 n d$, the $4 t h$ and the 5 th equation of the system (2.1), we remark that

$$
\frac{d \mathbf{G}}{d t}=\mathbf{F}^{2}(\mathbf{U})=\left[\begin{array}{c}
\left(1-f_{1}\right) K_{h} u_{1}+\left(1-f_{2}\right) \frac{1-Y}{Y}\left(\frac{d u_{2}}{d t}+K_{d} u_{2}\right) \\
f_{2} \frac{1-Y}{Y}\left(\frac{d u_{2}}{d t}+K_{d} u_{2}\right)
\end{array}\right] .
$$

This remark will be used in the section (3.2) in order to linearize the system (11).

\subsection{The flow system}

The waste is considered as a porous medium consisting of a solid matrix, a liquid phase (leachate) and a gaseous phase formed by a binary mixture of methane and carbon dioxide (biogas). In this article, we neglect the biogas flow and consider only the leachate flow 
which is governed by the generalized Darcy's Law (low Reynolds number) and the mass conservation equation. The equations are given as follows [35]

$$
\begin{array}{ll}
\frac{\partial(\rho \theta(p))}{\partial t}+\operatorname{div}(\rho \mathbf{u})=\alpha_{l} & \text { in } \quad \Omega \times] 0, T[, \\
\mathbf{u}=\frac{k_{i} k_{r}(p)}{\mu} \rho g\left(-\nabla p+e_{z}\right) & \text { in } \quad \Omega \times] 0, T[
\end{array}
$$

or

$$
\left.\frac{\partial(\rho \theta(p))}{\partial t}+\operatorname{div}\left(\rho \frac{k_{i} k_{r}(p)}{\mu} \rho g\left(-\nabla p+e_{z}\right)\right)=\alpha_{l} \quad \text { in } \quad \Omega \times\right] 0, T[
$$

where $\mathbf{u}$ is the velocity of the leachate, $k_{i}$ its intrinsic permeability, $k_{r}$ the relative permeability of leachate, $p$ its pressure head, $\mu$ the dynamic viscosity, $\rho$ the density, $g$ the modulus of the gravitational acceleration, $z$ the vertical axis directed downwards, $\theta$ the water content of the leachate and $\alpha_{l}$ is the source/sink term of leachate related to the rate of the biogas generation noted $\alpha_{b}$ which will be described later. There are many empirical models to describe the variation of the water content $\theta(p)$ (called the retention curve) and the relative permeability $k_{r}(p)$ in terms of the pressure head $p$ (see the Van Genuchten, Campbel and Brooks Corey model in [39], [12], [11]). We use here the Campbell model given by [12]

$$
\theta(p)= \begin{cases}\theta_{s}\left(\frac{p_{a}}{p}\right)^{1 / b} & \text { if } p<p_{a} \\ \theta_{s} & \text { if } p \geq p_{a}\end{cases}
$$

where $\theta_{s}$ is the saturation water content and $p_{a}$ is a scaling factor. $\left(\theta \approx \theta_{s}\right)$, and

$$
k_{r}(p)= \begin{cases}\left(\frac{p_{a}}{p}\right)^{2+3 / b} & \text { if } p<p_{a} \\ K_{s} & \text { if } p \geq p_{a}\end{cases}
$$

where $K_{s}$ is the saturation permeability, and $b$ is a parameter describing the uniformity of the grain size (see [29], [3], [38]).

SOURCE/SINK TERM

The production of the biogas needs water, so we have a sink term in equation (2.12) related to $\alpha_{b}$. We follow the approach of [3], [7], [1], [28]:

$$
\alpha_{l}=-\gamma \cdot \alpha_{b}=-\gamma g(\omega) C_{T b} \lambda_{\text {cin }}
$$

where $\gamma=M_{\mathrm{H}_{2} \mathrm{O}} /\left(3.4 * M_{b}\right)$ with $M_{\mathrm{H}_{2} \mathrm{O}}$ and $M_{b}$ are respectively the molar mass of $\mathrm{H}_{2} \mathrm{O}$ and of the biogas, $\omega$ is the humidity rate

$$
\theta=\frac{\rho_{0}}{\rho} \omega
$$

where $\rho_{0}$ is the density of the dry medium. $C_{T b}$ is a positive constant, and $\lambda_{\text {cin }}$ is the rate of the biogas production (see [20] and [22]) :

$$
\lambda_{\text {cin }}=\frac{d C_{\text {biogaz }}}{d t}=\frac{d\left(\left[\mathrm{CH}_{4}\right]+\left[\mathrm{CO}_{2}\right]\right)}{d t} .
$$


$g$ is an explicit function on the variable $\omega$ : the humidity rate. Experimental studies (see [3], [23]) show that below a minimal value $\omega_{\min }$ and above a maximum value $\omega_{\max }$ there is no biogas production. Between these two bounds, it increases, respectively decreases, linearly except on an interval where it is constant (that we normalize to 1 ), and where the medium is considered as saturated (see FIGURE (2)).

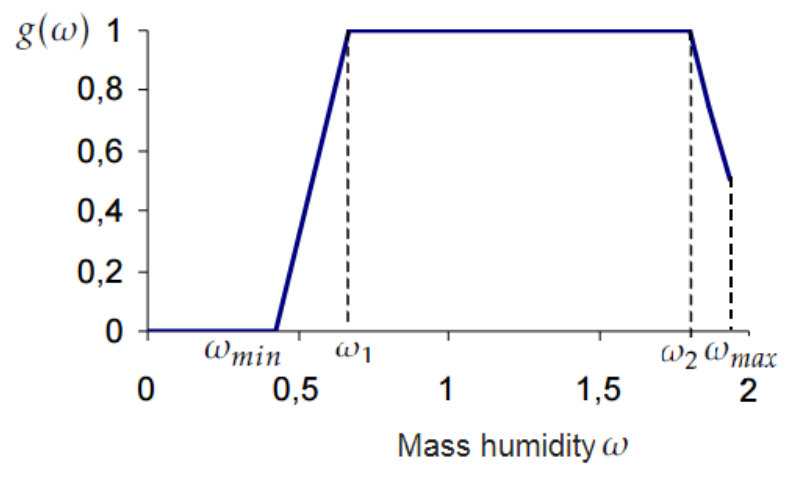

Figure 2: The empirical function $\tilde{g}(\omega)$.

\section{BOUNDARY AND INITIAL CONDITIONS}

The boundary conditions associated with the system (2.12)-(2.13) are expressed in term of charges (Dirichlet conditions) on $\Gamma_{D}$ :

$$
\left.p=p_{D} \quad \text { on } \quad \Gamma_{D} \times\right] 0, T[
$$

and in term of flux (Neumann conditions) on $\Gamma_{N}$ :

$$
\text { u.n } \left.=0 \quad \text { on } \quad \Gamma_{N} \times\right] 0, T[.
$$

The initial condition is given by

$$
p(x, 0)=p_{0}(x) \quad \text { in } \quad \Omega .
$$

We introduce the capillary capacity $\mathscr{C}(p)$ by rewriting the time derivative of the water content as follows

$$
\frac{\partial \theta}{\partial t}=\frac{\partial \theta}{\partial p} \frac{\partial p}{\partial t}=\mathscr{C}(p) \frac{\partial p}{\partial t}
$$

and by reversing the relative permeability $k_{r}$ and the density $\rho$, we set

$$
k(p)=\frac{\mu_{l}}{k_{i} \rho g}\left(k_{r}(p)\right)^{-1} \quad \text { and } \quad f=\rho^{-1} \alpha_{l} .
$$


The flow problem of leachate can be written finally as follows:

$$
\left\{\begin{aligned}
\mathscr{C}(p) \frac{\partial p}{\partial t}+\operatorname{div} \mathbf{u} & =f & & \text { in } \Omega \times] 0, T[, \\
k(p) \mathbf{u} & =-\nabla p+e_{z} & & \text { in } \Omega \times] 0, T[, \\
p & =p_{D} & & \text { on } \left.\Gamma_{D} \times\right] 0, T[, \\
\mathbf{u . n} & =0 & & \text { on } \left.\Gamma_{N} \times\right] 0, T[, \\
p(x, 0) & =p_{0}(x) & & \text { in } \Omega .
\end{aligned}\right.
$$

Solving this problem under mixed formulation is more suited in our case than solving Richards equation where the velocity is eliminated. In fact, it allows us to have more accuracy for $\mathbf{u}$ and in following the leachate variation during the production of biogas. Therefore, the unknowns in this formulation are both the velocity $\mathbf{u}$ and the pressure $p$.

\subsection{The final Model}

The final mathematical model describing the degradation of the organic mater and the leachate flow during the anerobic process is given by:

$(S 1) \quad \begin{cases}\frac{\partial \mathbf{U}}{\partial t}-\operatorname{div}(\overline{\bar{D}} \cdot \nabla \mathbf{U})=\mathbf{F}^{\mathbf{1}}(\mathbf{U}) & \text { in } \Omega \times] 0, T[, \\ \frac{d \mathbf{G}}{d t}=\mathbf{F}^{2}(\mathbf{U}) & \text { in } \Omega \times] 0, T[, \\ \overline{\bar{D}} \frac{\partial \mathbf{U}}{\partial \mathbf{n}}=0 & \text { on } \partial \Omega \times] 0, T[, \\ \mathbf{U}(0, \cdot)=\mathbf{U}^{0}(\cdot), \quad \mathbf{G}(0, \cdot)=\mathbf{G}^{0}(\cdot) & \text { in } \Omega,\end{cases}$

and

$$
\left\{\begin{aligned}
\mathscr{C}(p) \frac{\partial p}{\partial t}+\operatorname{div} \mathbf{u} & =f & & \text { in } \Omega \times] 0, T[, \\
k(p) \mathbf{u} & =-\nabla p+e_{z} & & \text { in } \Omega \times] 0, T[, \\
p & =p_{D} & & \text { on } \left.\Gamma_{D} \times\right] 0, T[, \\
\mathbf{u . n} & =0 & & \text { on } \left.\Gamma_{N} \times\right] 0, T[, \\
p(x, 0) & =p_{0}(x) & & \text { in } \Omega .
\end{aligned}\right.
$$




\section{Variational formulation and semi-discrete system}

In what follows, We make some assumptions:

Hypothesis 3.1. Coefficients $\alpha, K_{d}, Y, f_{1}$ and $f_{2}$ fulfill the following conditions.

1. The proportion of nutrient recycling $\alpha$ cannot exceed 1

$$
0<\alpha \leq 1
$$

2. The mortality rate $K_{d}$ is a positive parameter which is below the maximum growth rate

$$
0<K_{d}<\max _{s} \mu(s)
$$

3. The rate of use of the substrate is a strictly positive parameter such that

$$
0<Y<1
$$

4. The stoichiometric coefficients parameters $f_{1}$ and $f_{2}$ are strictly positive and satisfy

$$
0<f_{1}<1 \text { and } 0<f_{2}<1 \text {. }
$$

Hypothesis 3.2. The functions $p \longrightarrow \theta(p), p \longrightarrow \mathscr{C}(p)=\frac{\partial \theta}{\partial p}$ and $p \longrightarrow k(p)$ are uniformly bounded, i.e. there exist nonnegative constants $c_{\theta}, C_{\theta}, c_{\theta}^{\prime}, C_{\theta}^{\prime}, c_{k}$ and $C_{k}$ such that

$$
0<c_{\theta} \leq \theta(p) \leq C_{\theta}, \quad 0<c_{p} \leq \mathscr{C}(p) \leq C_{p}, \quad 0<c_{k} \leq k(p) \leq C_{k}
$$

The final model obtained in (2.22)-(2.23) is weakly coupled, therefore, we can separate the analysis of the system (2.22) and (2.23).

\subsection{Existence and uniqueness for problems $(S 1)-S(2)$}

We recall the definitions and notations used in this section. The usual Sobolev space

$$
H^{1}(\Omega)=\left\{v \in L^{2}(\Omega) \quad / \quad \nabla v \in\left(L^{2}(\Omega)\right)^{d}\right\},
$$

and the trace operator $\gamma_{0}: H^{1}(\Omega) \longrightarrow L^{2}(\Gamma)$. The trace space is

$$
H^{1 / 2}(\Gamma):=\gamma_{0}\left(H^{1}(\Omega)\right)
$$

and if $\Gamma_{D}$ is a part of the boundary of $\Omega$ we denote

$$
H_{\Gamma_{D}}^{1}(\Omega)=\left\{\phi \in H^{1}(\Omega), \quad \phi=0 \quad \text { on } \Gamma_{D}\right\} .
$$


We recall also that $H^{-1}(\Omega)$ is the dual of $H_{0}^{1}(\Omega)$. The space $L^{2}\left(0, T ; H^{1}(\Omega)\right)$ is the set of measurable functions

$u:] 0, T\left[\longrightarrow H^{1}(\Omega)\right.$ such that $\int_{0}^{T}\|u(t)\|_{H^{1}(\Omega)}^{2} d t<+\infty$, equipped with the norm

$$
\|u\|_{L^{2}\left(0, T ; H^{1}(\Omega)\right)}=\left[\int_{0}^{T}\|u(t)\|_{H^{1}(\Omega)}^{2} d t\right]^{1 / 2} .
$$

$L^{\infty}\left(Q_{T}\right)$ is the space of measurable functions $\left.u:\right] 0, T[\times \Omega \longrightarrow \mathbb{R}$ such that $|u(t, x)|$ is bounded almost everywhere in $Q_{T}$, equipped with the norm

$$
\|u\|_{L^{\infty}\left(Q_{T}\right)}=\inf \left\{C, \quad|u(t, x)|<C \quad \text { in } \quad Q_{T}\right\} .
$$

Finally, $C\left([0, T], L^{2}(\Omega)\right)$ is the space of continuous functions from $[0, T]$ with values in $L^{2}(\Omega)$ and $L^{\infty}\left(\Omega, \mathbb{R}_{+}^{3}\right)$ is the space of positive measurable functions $u: x \in \Omega \longrightarrow u(x) \in \mathbb{R}_{+}^{3}$ such that $u_{i}(x)$ is bounded almost everywhere in $\Omega$.

Due to the fact that the specific growth rate $\mu(\cdot)$ is a nonnegative and bounded function in both Monod and Haldane cases, and under hypothesis (3.1)-(3.2), it is readily checked that assumptions of Theorem 1 in [9] are verified and we have the following proposition.

Proposition 3.1. For any $\mathrm{U}^{0} \in L^{\infty}\left(\Omega, \mathbb{R}_{+}^{3}\right)$, the system (2.10) has a unique global nonnegative weak solution $\mathbf{U}=\left(u_{1}, u_{2}, u_{3}\right)^{T}$ in the following sense : $\forall T>0$

$$
\left.\begin{array}{l}
\forall i \in\{1,2,3\}, \quad u_{i} \in C\left([0, T] ; L^{2}(\Omega)\right) \cap L^{\infty}\left(Q_{T}\right) \cap L^{2}\left(0, T ; H^{1}(\Omega)\right) ; \\
\forall \psi \in C^{\infty}\left(Q_{T}\right) \text { such that } \psi(T)=0 \\
-\int_{\Omega} u_{i}^{0} \psi(0) d x-\int_{Q_{T}} u_{i} \frac{\partial \psi}{\partial t} d x d t+\int_{Q_{T}} D_{i} \nabla u_{i} \cdot \nabla \psi d x d t= \\
\int_{Q_{T}} F_{i}(\mathbf{U}(t, x)) \cdot \psi d x d t
\end{array}\right\}
$$

Moreover, for any $T>0$, there exists $C>0$ such that

$$
\|\mathbf{U}\|_{L^{\infty}\left(Q_{T}\right)^{3}}+\|\mathbf{U}\|_{L^{2}\left(0, T ; H^{1}(\Omega)^{3}\right)}+\left\|\partial_{t} \mathbf{U}\right\|_{L^{2}\left(0, T ; H^{-1}(\Omega)^{3}\right)} \leq C .
$$

Next, eliminating the velocity $\mathbf{u}$ in the Darcy equations, we obtain the well-known Richards equation on the pressure $p((2.14))$. The existence and uniqueness for this parabolic $\mathrm{PDE}$ is obtained in [2]. More precisely, we have

Proposition 3.2. For all $p^{0} \in L^{2}(\Omega)$ and $p_{D} \in H^{\frac{1}{2}}\left(\Gamma_{D}\right)$, There exists a unique weak solution $p-p_{D} \in L^{2}\left(0, T ; H_{\Gamma_{D}}^{1}(\Omega)\right)$ of the Richards equation (2.14). 


\subsection{Semi-discretization in time and variational formulation of the system (S1)}

Let $\tau_{n}=t^{n+1}-t^{n}, n=0, \ldots, N$, where $N \in \mathbb{N}^{*}$, be a partition of $[0, T]$ with $t^{0}=0$ and $t^{N}=T$. In what follows we denote by $f^{n}$ the values of a function $f$ at $t_{n}$.

The semi-discrete scheme for the system (S1) considered here is the second order backward differentiation formula BDF2 for the variables $(X, S, B)$ and the implicit Euler scheme for the ODEs on $\left(\left[\mathrm{CO}_{2}\right],\left[\mathrm{CH}_{4}\right]\right)$. The problem reads: find $\mathbf{U}^{n+1} \in Z=\left(\mathrm{H}^{1}(\Omega)\right)^{3}$ such that

$$
\begin{array}{r}
\int_{\Omega} \frac{3 \mathbf{U}^{n+1}-4 \mathbf{U}^{n}+\mathbf{U}^{n-1}}{2 \tau_{n}} \cdot \mathbf{v} d x+\int_{\Omega} \overline{\bar{D}} \nabla \mathbf{U}^{n+1} \cdot \nabla \mathbf{v} d x= \\
\int_{\Omega} \mathbf{F}^{1}\left(\mathbf{U}^{n+1}\right) \cdot \mathbf{v} d x \quad \forall \mathbf{v} \in Z
\end{array}
$$

where the initial values are computed as follow: find $\mathbf{U}^{1} \in Z$ such that

$$
\int_{\Omega} \frac{\mathbf{U}^{1}-\mathbf{U}^{0}}{\tau_{0}} \cdot \mathbf{v} d x+\int_{\Omega} \overline{\bar{D}} \cdot \nabla \mathbf{U}^{1} \cdot \nabla \mathbf{v} d x=\int_{\Omega} \mathbf{F}^{\mathbf{1}}\left(\mathbf{U}^{1}\right) \cdot \mathbf{v} d x \quad \forall \mathbf{v} \in Z .
$$

We linearize the scheme by using the Taylor formula

$$
\mathbf{F}^{\mathbf{1}}\left(\mathbf{U}^{n+1}\right) \simeq \mathbf{F}^{\mathbf{1}}\left(\mathbf{U}^{n}\right)+J_{\mathbf{F}^{1}}\left(\mathbf{U}^{n}\right) \cdot\left(\mathbf{U}^{n+1}-\mathbf{U}^{n}\right)
$$

where $J_{\mathrm{F}^{1}}$ is the Jacobian matrix of the vector function $\mathrm{F}^{\mathbf{1}}$. Consequently, the final BDF2 scheme reads : find $\mathbf{U}^{1} \in Z=\left(H^{1}(\Omega)\right)^{3}$ such that

$$
\begin{gathered}
\int_{\Omega}\left[\tau_{0}^{-1} \mathbf{I}_{3}-J_{\mathrm{F}^{1}}\left(\mathbf{U}^{0}\right)\right] \cdot \mathbf{U}^{1} \cdot \mathbf{v} d x+\int_{\Omega} \overline{\bar{D}} \cdot \nabla \mathbf{U}^{1} \cdot \nabla \mathbf{v} d x= \\
\int_{\Omega}\left[\tau_{0}^{-1} \mathbf{U}^{0}+\mathbf{F}^{1}\left(\mathbf{U}^{0}\right)-J_{\mathrm{F}^{1}}\left(\mathbf{U}^{0}\right) \cdot \mathbf{U}^{0}\right] \cdot \mathbf{v} d x \quad \forall \mathbf{v} \in Z,
\end{gathered}
$$

and for all $n \geq 1$, find $\mathbf{U}^{n+1} \in Z$ such that

$$
\begin{gathered}
\int_{\Omega}\left[\frac{3}{2 \tau_{n}} \mathbf{I}_{3}-J_{\mathbf{F}^{1}}\left(\mathbf{U}^{n}\right)\right] \cdot \mathbf{U}^{n+1} \cdot \mathbf{v} d x+\int_{\Omega} \overline{\bar{D}} \cdot \nabla \mathbf{U}^{n+1} \cdot \nabla \mathbf{v} d x= \\
\int_{\Omega}\left[\frac{4 \mathbf{U}^{n}-\mathbf{U}^{n-1}}{2 \tau_{n}}+\mathbf{F}^{\mathbf{1}}\left(\mathbf{U}^{n}\right)-J_{\mathbf{F}^{1}}\left(\mathbf{U}^{n}\right) \cdot \mathbf{U}^{n}\right] \cdot \mathbf{v} d x \quad \forall \mathbf{v} \in Z .
\end{gathered}
$$


On the other hand, by using the Remark 1, the approximation of $\mathbf{G}$ at $t=t_{m+1} \mathbf{G}^{m+1}=$ $\left(u_{4}^{m+1}, u_{5}^{m+1}\right)^{T}$, for, $0 \leq m \leq n$, is given by

$$
\begin{array}{r}
\frac{u_{4}^{m+1}-u_{4}^{m}}{\tau_{m}}=\left(1-f_{1}\right) K_{h} u_{1}^{m+1}+\left(1-f_{2}\right) \frac{1-Y}{Y}\left(\frac{u_{2}^{m+1}-u_{2}^{m}}{\tau_{m}}+K_{d} u_{2}^{m+1}\right), \\
\frac{u_{5}^{m+1}-u_{5}^{m}}{\tau_{m}}=f_{2} \frac{1-Y}{Y}\left(\frac{u_{2}^{m+1}-u_{2}^{m}}{\tau_{m}}+K_{d} u_{2}^{m+1}\right) .
\end{array}
$$

\subsection{Semi-discretization in time and variational formulation of the system (S2)}

We use the semi-implicit scheme for (S2) :

$$
\begin{aligned}
k\left(p^{n}\right) \mathbf{u}^{n+1}=-\nabla p^{n+1}+e_{z} & \text { in } \Omega, \\
\mathscr{C}\left(p^{n}\right) \frac{p^{n+1}-p^{n}}{\tau_{n}}+\operatorname{div} \mathbf{u}^{n+1}=f^{n+1} & \text { in } \Omega,
\end{aligned}
$$

where the unknowns $\mathbf{u}^{n+1}$ and $p^{n+1}$ refer to the approximation of $\mathbf{u}\left(t_{n+1}\right)$ and $p\left(t_{n+1}\right)$ with

$$
\mathbf{u}^{n+1} \cdot \mathbf{n}=0 \text { on } \Gamma_{N} \quad \text { and } \quad p^{n+1}=p_{D} \text { on } \Gamma_{D} .
$$

MIXED VARIATIONAL FORMULATION

We introduce the following functional spaces

$$
\begin{aligned}
H(\operatorname{div}, \Omega) & =\left\{\mathbf{v} \in\left(L^{2}(\Omega)\right)^{d}, \quad \operatorname{div} \mathbf{v} \in L^{2}(\Omega)\right\}, \\
\mathbf{V} & =H_{0, N}(\Omega)=\left\{\mathbf{v} \in H(\operatorname{div}, \Omega), \quad \mathbf{v . n}=0 \text { on } \Gamma_{N}\right\}, \\
W & =L^{2}(\Omega),
\end{aligned}
$$

equipped with the norms

$$
\forall \mathbf{v} \in \mathbf{V}, \quad\|\mathbf{v}\|_{\mathbf{v}}=\|\mathbf{v}\|_{H(\operatorname{div}, \Omega)}=\left(\|\mathbf{v}\|_{0, \Omega}^{2}+\|\operatorname{div} \mathbf{v}\|_{0, \Omega}^{2}\right)^{1 / 2}
$$

and

$$
\forall \varphi \in W, \quad\|\varphi\|_{W}=\|\varphi\|_{0, \Omega}
$$

where $\|\cdot\|_{0, \Omega}$ is the $L^{2}$-norm and we denote the dual spaces of $\mathbf{V}$ and $W$ by $\mathbf{V}^{\prime}$ and $W^{\prime}$ respectively. The weak formulation of (3.15)-(3.16) with the conditions (3.17) is now written as follows:

$$
\left\{\begin{array}{l}
\int_{\Omega}^{\text {find } \mathbf{u}^{n+1} \in \mathbf{V} \text { and } p^{n+1} \in W \text { such that }} k\left(p^{n}\right) \mathbf{u}^{n+1} \cdot \psi d x-\int_{\Omega} \operatorname{div} \psi p^{n+1}=-\int_{\Gamma_{D}} p_{D} \psi \cdot \mathbf{n} d s+\int_{\Omega} e_{z} \cdot \psi d x, \quad \forall \psi \in \mathbf{V}, \\
\int_{\Omega} \operatorname{div} \mathbf{u}^{n+1} \varphi d x+\int_{\Omega} \frac{\mathscr{C}\left(p^{n}\right)}{\tau_{n}} p^{n+1} \varphi d x=\int_{\Omega} \frac{\mathscr{C}\left(p^{n}\right)}{\tau_{n}} p^{n} \varphi d x+\int_{\Omega} f^{n+1} \varphi d x, \quad \forall \varphi \in W,
\end{array}\right.
$$


which can be written in the following form

$$
\begin{cases}\text { find }\left(\mathbf{u}^{n+1}, p^{n+1}\right) \in \mathbf{V} \times W \text { such that } & \\ a\left(\mathbf{u}^{n+1}, \psi\right)+b\left(\psi, p^{n+1}\right)=g_{1}(\psi), & \forall \psi \in \mathbf{V}, \\ b\left(\mathbf{u}^{n+1}, \varphi\right)-d\left(p^{n+1}, \varphi\right)=g_{2}(\varphi), & \forall \varphi \in W,\end{cases}
$$

where $a: \mathbf{V} \times \mathbf{V} \longrightarrow \mathbb{R}, b: \mathbf{V} \times W \longrightarrow \mathbb{R}$ and $d: W \times W \longrightarrow \mathbb{R}$ are the bilinear forms defined by

$$
\begin{aligned}
& a(\mathbf{u}, \psi)=\int_{\Omega} k\left(p^{n}\right) \mathbf{u} \cdot \psi d x \quad ; \quad(\mathbf{u}, \psi) \in \mathbf{V} \times \mathbf{V} \\
& b(\mathbf{u}, \varphi)=-\int_{\Omega} \operatorname{div} \mathbf{u} \varphi ; \quad(\mathbf{u}, \varphi) \in \mathbf{V} \times W \\
& d(p, \varphi)=\int_{\Omega} \frac{\mathscr{C}\left(p^{n}\right)}{\tau_{n}} p \varphi d x \quad ; \quad(p, \varphi) \in W \times W
\end{aligned}
$$

and the second members $g_{1}: \mathbf{V} \longrightarrow \mathbb{R}$ and $g_{2}: W \longrightarrow \mathbb{R}$ are the linear forms defined by

$$
\begin{aligned}
& g_{1}(\psi)=-\int_{\Gamma_{D}} p_{D} \psi \cdot \mathbf{n} d s+\int_{\Omega} e_{z} \cdot \psi d x \quad ; \quad \psi \in \mathbf{V} \\
& g_{2}(\varphi)=-\int_{\Omega} \frac{\mathscr{C}\left(p^{n}\right)}{\tau_{n}} p^{n} \varphi d x-\int_{\Omega} f^{n+1} \varphi d x ; \quad \varphi \in W .
\end{aligned}
$$

We define the continuous operator $B: \mathbf{u} \in \mathbf{V} \longrightarrow \operatorname{div} \mathbf{u} \in W^{\prime}$ and its transpose $B^{t}: W \longrightarrow \mathbf{V}^{\prime}$ such that

$$
<B \mathbf{v}, \varphi>_{W^{\prime} \times W}=<\mathbf{v}, B^{t} \varphi>_{\mathbf{v}^{\prime} \times \mathbf{v}}=b(\mathbf{v}, \varphi) \quad \forall \mathbf{v} \in \mathbf{V}, \forall \varphi \in W,
$$

and then we introduce the subspace of $\mathbf{V}$ :

$$
\operatorname{Ker} B=\{\mathbf{v} \in \mathbf{V}, \quad \forall \varphi \in W, \quad b(\mathbf{v}, \varphi)=0\} .
$$

\subsubsection{Existence and uniqueness of the solution of (3.19)}

Proposition 3.3. For all $p_{D} \in H^{1 / 2}\left(\Gamma_{D}\right)$ and $f \in L^{2}(\Omega)$ the system (3.19) has a unique solution, and we have the estimate

$$
\left\|\boldsymbol{u}^{n}\right\|_{\mathrm{v}} \leq C_{1}\left\|g_{1}\right\|_{\mathbf{v}^{\prime}}+C_{2}\left\|g_{2}\right\|_{W^{\prime}} \text { and }\left\|p^{n}\right\|_{W} \leq C_{2}\left\|g_{1}\right\|_{\mathbf{v}^{\prime}}+C_{3}\left\|g_{2}\right\|_{W^{\prime}}
$$


with

$$
\begin{gathered}
C_{1}=\frac{\left(1+C_{\Omega}^{2}\right)^{-1}+4 \tau_{n-1}^{-1} C\left(p^{n-1}\right) C_{k}}{c_{k}\left(1+C_{\Omega}^{2}\right)^{-1}} \quad, \quad C_{2}=\frac{2 C_{k}^{1 / 2}}{c_{k}^{1 / 2}\left(1+C_{\Omega}^{2}\right)^{-1 / 2}}, \\
C_{3}=\frac{4 C_{k}}{\tau_{n-1}^{-1} C\left(p^{n-1}\right)+2\left(1+C_{\Omega}^{2}\right)^{-1}}
\end{gathered}
$$

where $C_{\Omega}$ is the Poincaré constant.

Proof. The bilinear form $a(\cdot, \cdot)$ is symmetric, continuous and KerB-elliptic according to the hypothesis (3.2) and since in $\operatorname{Ker} B$, the norm (3.18) is reduced to the $L^{2}$-norm. The bilinear form $d(\cdot, \cdot)$ is symmetric, continuous and $W$-elliptic

$$
\begin{aligned}
|d(p, \varphi)| & \leq \gamma_{1}\|p\|_{W}\|\varphi\|_{W} \quad \forall(p, \varphi) \in W \times W, \\
d(\varphi, \varphi) & \geq \gamma_{2}\|\varphi\|_{W}^{2} \quad \forall \varphi \in W, \\
\text { with } \gamma_{1} & =\frac{C_{p}}{\tau_{n}} \text { and } \gamma_{2}=\frac{c_{p}}{\tau_{n}} .
\end{aligned}
$$

Moreover, the bilinear form $b(\cdot, \cdot)$ is continue on $\mathbf{V} \times W$, and applying Lax-Milgram theorem to the auxiliary problem

$$
\left\{\begin{array}{l}
-\Delta \mathbf{v}=\varphi \text { in } \Omega \\
\mathbf{v}=0 \text { on } \Gamma_{D} \\
\nabla \mathbf{v} \cdot \mathbf{n}=0 \text { on } \Gamma_{N}
\end{array}\right.
$$

we deduce in standard way that $b(\cdot, \cdot)$ satisfies the inf - sup condition (e.g. [8])

$$
\exists \beta=\frac{1}{\sqrt{1+C_{\Omega}^{2}}}>0 ; \quad \forall \varphi \in W, \quad \sup _{\mathbf{u} \in \mathbf{V}} \frac{b(\mathbf{u}, \varphi)}{\|\mathbf{u}\|_{\mathrm{V}}} \geq \beta\|\varphi\|_{W} .
$$

Therefore, the existence follows from Brezzi-Babuska theorem.

\section{Discrete problem and analysis}

We assume in this section that $\Omega$ is a polygonal domain of $\mathbb{R}^{d}$. Let $\mathscr{T}_{h}$ be a partition of $\bar{\Omega}$ into triangles $T$ (in $\mathbb{R}^{2}$ ) or tetrahedra (in $\mathbb{R}^{3}$ ) and we denote by $P_{k}(O)$ the space of polynomial functions defined in a subset $O$ of $\mathbb{R}^{d}$ of total degree at most $k$. In order to define the full discritization of the problem (2.22)-(2.23), we introduce the following finite-dimensional space

$$
Z_{h}=\left\{\mathbf{v}_{h} \in(C(\bar{\Omega}))^{3} ;\left.\forall T \in \mathscr{T}_{h} \quad \mathbf{v}_{h}\right|_{T} \in\left(P_{1}(T)\right)^{3}\right\} .
$$

We recall the definition of the local Raviart-Thomas space of lowest order (see [10]), $\forall T \in$ $\mathscr{T}_{h}$

$$
R T_{0}(T):=\left\{\mathbf{v}_{h} \in H(\operatorname{div}, T): \quad \mathbf{v}_{h} \in P_{0}^{d}(T) \oplus \mathbf{x} P_{0}(T), \quad \mathbf{x} \in T\right\},
$$


and the global Raviart-Thomas space of lowest order is given by

$$
R T_{0}\left(\mathscr{T}_{h}\right):=\left\{\mathbf{v}_{h} \in H(\operatorname{div}, \Omega):\left.\quad \mathbf{v}_{h}\right|_{T} \in R T_{0}(T), \forall T \in \mathscr{T}_{h}\right\}
$$

We introduce the discrete spaces

$$
\mathbf{V}_{h}=\left\{\mathbf{v}_{h} \in R T_{0}\left(\mathscr{T}_{h}\right) \quad \mathbf{v}_{h} \cdot \mathbf{n}_{\mid \Gamma_{N}}=0\right\} .
$$

and

$$
W_{h}:=\left\{q_{h} \in L^{2}(\Omega):\left.\quad q_{h}\right|_{T} \in P_{0}(T), \forall T \in \mathscr{T}_{h}\right\} .
$$

\subsection{Full discretization of the system (S1)}

The discrete problem associated to (3.11)-(3.14) reads : find $\mathbf{U}_{h}^{1} \in Z_{h}$ such that

$$
\begin{gathered}
\int_{\Omega}\left[\tau_{0}^{-1} \mathbf{I}_{3}-J_{\mathrm{F}^{1}}\left(\mathbf{U}^{0}\right)\right] \cdot \mathbf{U}_{h}^{1} \cdot \mathbf{v} d x+\int_{\Omega} \overline{\bar{D}} \cdot \nabla \mathbf{U}_{h}^{1} \cdot \nabla \mathbf{v} d x= \\
\int_{\Omega}\left[\tau_{0}^{-1} \mathbf{U}^{0}+\mathbf{F}^{1}\left(\mathbf{U}^{0}\right)-J_{\mathbf{F}^{1}}\left(\mathbf{U}^{0}\right) \cdot \mathbf{U}^{0}\right] \cdot \mathbf{v} d x \quad \forall \mathbf{v} \in Z_{h},
\end{gathered}
$$

and for all $n \geq 1$, find $\mathbf{U}_{h}^{n+1} \in Z_{h}$ such that

$$
\begin{gathered}
\int_{\Omega}\left[\frac{3}{2 \tau_{n}} \mathbf{I}_{3}-J_{\mathbf{F}^{1}}\left(\mathbf{U}_{h}^{n}\right)\right] \cdot \mathbf{U}_{h}^{n+1} \cdot \mathbf{v} d x+\int_{\Omega} \overline{\bar{D}} \cdot \nabla \mathbf{U}_{h}^{n+1} \cdot \nabla \mathbf{v} d x= \\
\int_{\Omega}\left[\frac{4 \mathbf{U}^{n}-\mathbf{U}^{n-1}}{2 \tau_{n}}+\mathbf{F}^{1}\left(\mathbf{U}^{n}\right)-J_{\mathbf{F}^{1}}\left(\mathbf{U}^{n}\right) \cdot \mathbf{U}^{n}\right] \cdot \mathbf{v} d x \quad \forall \mathbf{v} \in Z_{h} .
\end{gathered}
$$

On the other hand, we seek $\mathbf{G}_{h}^{m+1}=\left(u_{4 h}^{m+1}, u_{5 h}^{m+1}\right)^{T}, \forall m, 0 \leq m \leq n$, such that

$$
\begin{array}{r}
\frac{u_{4 h}^{m+1}-u_{4 h}^{m}}{\tau_{m}}=\left(1-f_{1}\right) K_{h} u_{1 h}^{n+1}+\left(1-f_{2}\right) \frac{1-Y}{Y}\left(\frac{u_{2 h}^{m+1}-u_{2 h}^{m}}{\tau_{m}}+K_{d} u_{2 h}^{m+1}\right), \\
\frac{u_{5 h}^{m+1}-u_{5 h}^{m}}{\tau_{m}}=f_{2} \frac{1-Y}{Y}\left(\frac{u_{2 h}^{m+1}-u_{2 h}^{m}}{\tau_{m}}+K_{d} u_{2 h}^{m+1}\right) .
\end{array}
$$

Remark 4.1. In practice we choose $m=n$ in our numerical simulation to solve in one step the discrete problem. Nevertheless, we write under this general form because it may be suitable for some applications two have to time grid for the ODEs part and the reactiondiffusion term. 


\subsection{Full discretization of the system (S2)}

The mixed finite element method formulation of (3.19) reads

$$
\left\{\begin{array}{l}
\text { find }\left(\mathbf{u}_{h}^{n+1}, p_{h}^{n+1}\right) \in \mathbf{V}_{h} \times W_{h} \text { such that } \\
a\left(\mathbf{u}_{h}^{n+1}, \psi_{h}\right)+b\left(\psi_{h}, p_{h}^{n+1}\right)=g_{1}\left(\psi_{h}\right) \forall \psi_{h} \in \mathbf{V}_{h} \\
b\left(\mathbf{u}_{h}^{n+1}, \varphi_{h}\right)-d\left(p_{h}^{n+1}, \varphi_{h}\right)=g_{2}\left(\varphi_{h}\right) \forall \varphi_{h} \in W_{h}
\end{array}\right.
$$

EXISTENCE AND UNIQUENESS FOR THE DISCRETE PROBLEMS

Proposition 4.1. Problem (4.10) admits a unique solution.

Proof. We denote $B_{h}: \mathbf{V}_{h} \longrightarrow W_{h}^{\prime}$ the operator

$$
b\left(\mathbf{v}_{h}, \varphi_{h}\right)=<B_{h} \mathbf{v}_{h}, \varphi_{h}>_{W_{h}^{\prime} \times W_{h}} \quad \forall \mathbf{v} \in \mathbf{V}_{h}, \forall \varphi_{h} \in W_{h}
$$

and we introduce the kernel of $B_{h}$ :

$$
\operatorname{Ker} B_{h}=\left\{\mathbf{v}_{h} \in \mathbf{V}_{h}, \forall \varphi_{h} \in W_{h}, b\left(\mathbf{v}_{h}, \varphi_{h}\right)=0\right\} .
$$

Let $P_{h}$ be the orthogonal projection operator from $W$ to $W_{h}$, then for all $\mathbf{v}_{h} \in \mathbf{V}_{h}$ and $q \in W$ Thus, $\operatorname{Ker}_{h} \subset \operatorname{Ker} B$, and $a(\cdot, \cdot)$ is uniformly $\left(\operatorname{Ker} B_{h}\right)$-elliptic: there exists a constant $\alpha_{0}$ independent of $h$ such

$$
a\left(\mathbf{u}_{h}, \mathbf{u}_{h}\right) \geq \alpha_{0}\left\|\mathbf{u}_{h}\right\|_{\mathbf{v}}^{2} \quad \forall \mathbf{u}_{h} \in \operatorname{Ker} B_{h} .
$$

Moreover, the discrete inf-sup condition follows from the continuous one. There exists a constant $\beta>0$ independent of $h$ such that

$$
\inf _{v_{h} \in \mathrm{V}_{h}} \sup _{w_{h} \in W_{h}} \frac{b\left(v_{h}, w_{h}\right)}{\left\|v_{h}\right\|_{V}\left\|w_{h}\right\|_{W}} \geq \beta
$$

We deduce the existence and uniqueness of the solution of (3.19) thanks to Brezzi-Babuska theorem.

\section{A PRIORI ESTIMATES}

In the sequel we consider (3.19) and (4.10) and we drop the subscript $n$ for simplicity.

Proposition 4.2. Let $(\boldsymbol{u}, p)$, respectively $\left(\boldsymbol{u}_{h}, p_{h}\right)$, denotes the solutions of problems (3.19), respectively (4.10). We assume that $\boldsymbol{u} \in\left(H^{2}(\Omega)\right)^{2}$ and $p \in H^{1}(\Omega)$, then there exists a constant $\mathrm{C}>0$ independent of $h$ which satisfies the following estimate

$$
\left\|\boldsymbol{u}-\boldsymbol{u}_{h}\right\|_{\mathbf{V}}+\left\|p-p_{h}\right\|_{W} \leq \mathbf{C h}\left(|\boldsymbol{u}|_{1, \Omega}+|\operatorname{div} \boldsymbol{u}|_{1, \Omega}+|p|_{1, \Omega}\right)
$$


Proof. Combining the ellipticity of the bilinear form $a(.,$.$) and the uniform discrete$ inf-sup condition on $b(.,$.$) it is standard to derive the abstract estimate$

$$
\left\|\mathbf{u}-\mathbf{u}_{h}\right\|_{\mathbf{V}}+\left\|p-p_{h}\right\|_{W} \leq \bar{C}\left(\inf _{\mathbf{v}_{h} \in V_{h}}\left\|\mathbf{u}-\mathbf{v}_{h}\right\|_{\mathbf{V}}+\inf _{q_{h} \in W_{h}}\left\|p-q_{h}\right\|_{W}\right) .
$$

Using the interpolation operators Raviart-Thomas operator $\Pi_{h}$ and the the orthogonal $L^{2}$ projection operator $P_{h}$, we get ( [24], [10])

$$
\left\|\mathbf{u}-\mathbf{u}_{h}\right\|_{\mathbf{V}}+\left\|p-p_{h}\right\|_{W} \leq \mathbf{C h}\left(|\mathbf{u}|_{1, \Omega}+|\operatorname{div} \mathbf{u}|_{1, \Omega}+|p|_{1, \Omega}\right) .
$$

\section{Numerical results}

In this section we present some numerical results for the system $\left(S_{1}\right)-\left(S_{2}\right)$. In all the experiments, we take $\Omega=] 0,1[\times] 0,1[\times] 0,1\left[\subset \mathbb{R}^{3}, \partial \Omega=\Gamma_{D} \cup \Gamma_{N}\right.$ and $\Gamma_{D}=\{(x, y) \in$ ]0, $1[\times] 0,1[$ and $z=1\}$ for 3D simulations and $\Omega=\{(x, y) \in] 0,1[\times] 0,1[$ and $z=0\}$, with the corresponding boundaries for 2D simulations. The various constants used are fixed as in [36] and [3] and given in TABLE (1), and from the same references, we take the initial conditions for the biodegradation system (in $\mathrm{mgC} / \mathrm{L}$ ) as given in TABLE (2). The boundary conditions for the biodegradation system are all homogeneous Neumann conditions (see (2.5)). The boundary and initial conditions for the flow system are given in TABLE (3).

We recall that, at each time step, the system $\left(S_{1}\right)$ is approximated by (4.6)-(4.9), and its solution gives the source term in the flow system $\left(S_{2}\right)$, approximated by (4.10).

Table 1: Parameters.

\begin{tabular}{||l|l|c|c|c|c|c|c|c|c|c|c||}
\hline \multicolumn{1}{c|}{} & $K_{H}$ & $\mu_{m}$ & $f_{1}$ & $f_{2}$ & $K_{S}$ & $K_{d}$ & $Y$ & $\alpha$ & $K_{I}$ & \multicolumn{2}{|c||}{$\mu_{l}$} \\
\cline { 2 - 11 } \multicolumn{1}{c|}{} & 0.176 & 0.3 & 0.7 & 0.76 & 160 & 0.04 & 0.05 & 0.9 & 500 & \multicolumn{2}{|c|}{$4.61027 \times 10^{-4}$} \\
\hline$\theta_{r}$ & $\theta_{s}$ & $k_{i}$ & $p_{a}$ & $b$ & $M_{b}(\mathrm{~g} / \mathrm{mol})$ & $M_{\mathrm{H}_{2} \mathrm{O}}(\mathrm{g} / \mathrm{mol})$ & $A_{m}$ & $C_{T b}\left(\mathrm{~m}^{3} / \mathrm{Kg}\right)$ \\
\hline 0.27 & 0.9715 & $10^{-4}$ & -0.0323 & 2.5 & \multicolumn{2}{c|}{30} & 18.01 & 0.8 & 0.178 \\
\hline
\end{tabular}

Table 2: Initial conditions for the biodegradation system.

\begin{tabular}{||l|c|c|c||}
\hline$X_{0}(x, y)$ & $S_{0}(x, y)$ & {$\left[\mathrm{CO}_{2}\right]_{0}(x, y)$} & {$\left[\mathrm{CH}_{4}\right]_{0}(x, y)$} \\
\hline 1751 & 0 & 0 & 0 \\
\hline
\end{tabular}

Table 3: Initial and boundary conditions for the flow system.

\begin{tabular}{||l|c|c||}
\hline$p_{0}$ in $\Omega$ & $p_{D}$ on $\Gamma_{D}$ & $\mathbf{u} \cdot \mathbf{n}$ on $\Gamma_{N}$ \\
\hline-0.7 & -0.1020 & 0 \\
\hline
\end{tabular}

The two first examples, less sensitive to the dimension, are 2D simulations for comparison purposes. The two last examples are the preliminary 3D simulations. Notice that in all 
the examples, except (5.4), we consider only the system $(S 1)$ for comparisons with existing results in the literature. This is not a restriction due to the weak coupling of (S1) and (S2) in the present model.

We first compare the numerical results obtained with the reaction-diffusion system $\left(S_{1}\right)$ and the ones obtained in [36] and in [31]. In example (5.2), we use different values of the diffusion coefficients in order to show the effect on the final solution. Example (5.3) consists of solving $\left(S_{1}\right)$ with the partial diffusion and with the complete diffusion (on all the variables of $(S 1))$. The last example consists of solving the full system $(S 1)-(S 2)$.

\subsection{Model verification}

In this example, we denote $\overline{\bar{D}}_{\text {val }}=\operatorname{diag}\left(D_{X}, D_{S}, D_{B}\right)$, with $D_{X}=0.1, D_{S}=0.3$ and $D_{B}=0.5$, the diffusion matrix. We set $B_{0}=2$ and we use Monod law, and we plot in FIGURE (3) the evolution in time of all the variables. We notice that the results are identical to similar numerical experiments in the literature for $(S 1)$ without diffusion (e.g. [36]). Next, we use Haladane law and we represent in FIGURE (4) the same curves but with different values of $X_{0}$, which also confirms the previous results. Moreover, in FIGURE (5) we plot the curve of the biogas [CH4]+[CO2] as a function of the initial value $X_{0}$ and we obtain a discontinuity, already noticed in [31]. In fact, if $X_{0}<475$ the curve is monotonically increasing otherwise, it is decreasing. The explanation of this behaviour of the dynamical system is thouroughly detailed in [31] and the added diffusion do not change qualitatively the expected dynamic. In particular, Haladane law, usually, considered as more realistic (and the Monod function can be seen as a particular case for large values of the parameter $K_{I}$, see FIGURE (6)) yields more accurate predictions on the biogas production.

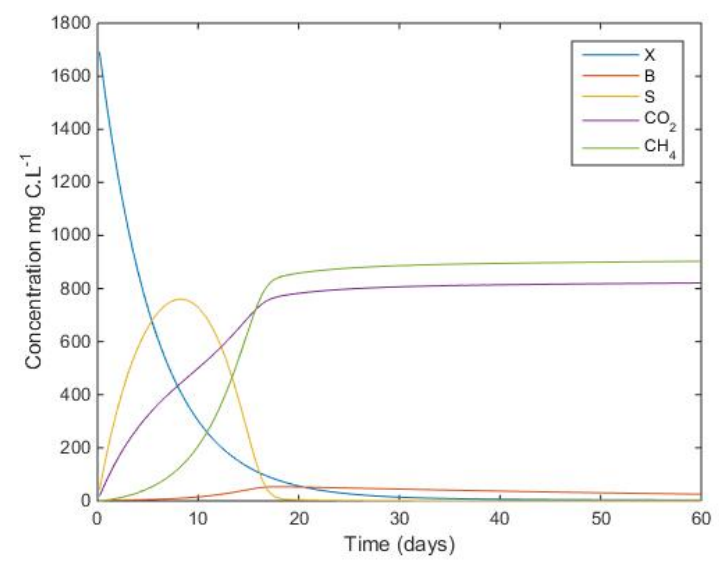

Figure 3: Evolution of the averages variables of (S1) using the Monod law and the diffusion $\overline{\bar{D}}_{\text {val }}$ and $B_{0}=2, X_{0}=1751, \alpha=1$ 


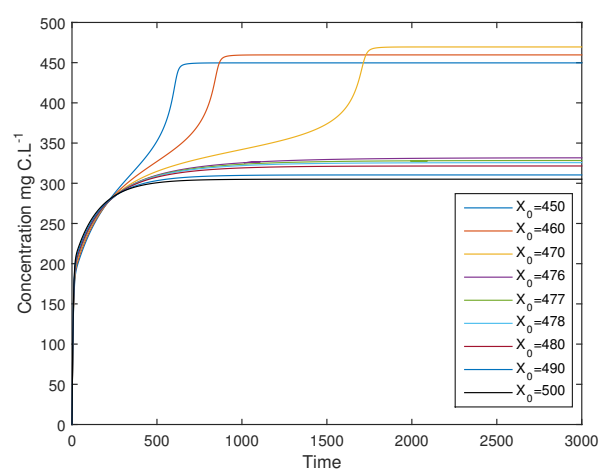

Figure 4: Evolution of the average quantity of biogas using the Haldane law with $B_{0}=2, K_{I}=10$ and the diffusion $D_{v a l}^{=}$for different values of $X_{0}$

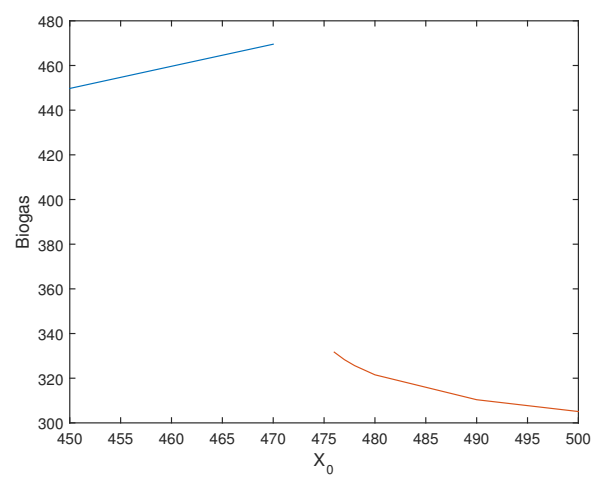

Figure 5: Biogas production as a function of Evolution of $X_{0}$ using the Haldane law with $B_{0}=2, K_{I}=10$ and the diffusion $D_{\text {val }}$

\subsection{Effect of the diffusion parameters on the biogas production}

In this example, we use and compare several choices of the diffusion coefficients on the solution of the reaction-diffusion system. We set

$$
\begin{aligned}
& \overline{\bar{D}}_{2}=\left\{D_{X}=0.001, \quad D_{S}=0.003, \quad D_{B}=0.005\right\} \\
& \overline{\bar{D}}_{3}=\left\{D_{X}=0.01, \quad D_{S}=0.03, \quad D_{B}=0.03\right\} \\
& \overline{\bar{D}}_{4}=\left\{D_{X}=0.1, \quad D_{S}=0.3, \quad D_{B}=0.5\right\}
\end{aligned}
$$

and we consider the initial condition on $B$ as follow

$$
B_{0}(x, y)=\left\{\begin{array}{lll}
0 & \text { in } & \sum_{1}:=\{0 \leq x \leq 0.5,0.5 \leq y \leq 1 \text { and } 0.5 \leq z \leq 1\}, \\
2 & \text { in } \quad \sum_{2}:=\{0.5 \leq x \leq 1,0.5 \leq y \leq 1 \text { and } 0.5 \leq z \leq 1\}, \\
5 & \text { in } \quad \sum_{3}:=\{0 \leq x \leq 1,0<y<0.5 \text { and } 0 \leq z<0.5\}
\end{array}\right.
$$

In TABLE (4), we observe that the values of the diffusion coefficients have a negligible effect on the average values of the biogas production. 


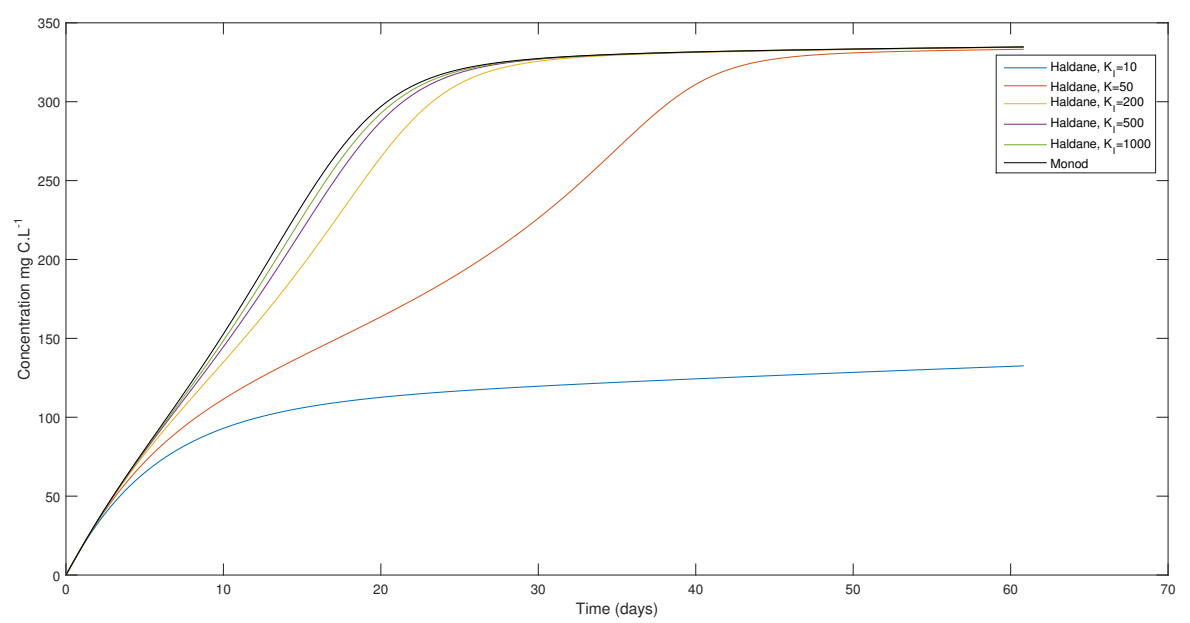

Figure 6: Evolution of all the average quantities of biogas production of (S1) with the diffusion $\overline{\bar{D}}_{\text {val }}$ and $X_{0}=340, B_{0}=2$ as in [31] and using the Monod and the Haldane law (for $K_{I}=10,50,200,500,1000$ )

Figures (7) and (8) show that the lack of methanogenic bacteria in a given area (i.e. $B_{0}=0$ in $\sum_{1}$ ), induces nul biogas production in $\Sigma_{1}$ and sharp transition between $\Sigma_{i}$, $i=1,2,3$ in the case of no diffusion, whereas with the additional diffusion, the transition become more diffuse and biogas production expand to the entire domain.

Table 4: Four days evolution of the average quantity of methane using different values of diffusion with the Haldane law.

\begin{tabular}{||l|cccc||}
\hline Diffusion $\overline{\bar{D}}_{i}$ & $\mathbf{0 . 5}$ day & $\mathbf{1}$ days & $\mathbf{1 . 5}$ days & 2 days \\
\hline$\overline{\bar{D}}_{0}$ (no diffusion term) & 1.60651 & 4.48873 & 7.89388 & $\mathbf{1 1 . 5 5 3 0}$ \\
$\overline{\bar{D}}_{2}$ & 1.60671 & 4.48927 & 7.89465 & $\mathbf{1 1 . 5 5 3 7}$ \\
$\overline{\bar{D}}_{3}$ & 1.60737 & 4.49098 & 7.89703 & $\mathbf{1 1 . 5 5 6 0}$ \\
$\overline{\bar{D}}_{4}$ & 1.60949 & 4.49647 & 7.90468 & $\mathbf{1 1 . 5 6 3 3}$ \\
\hline
\end{tabular}

\subsection{Comparison of the partial diffusion $\overline{\bar{D}}$ and the complete diffusion $\overline{\bar{D}}^{c}$.}

In this example, we compare the partial diffusion with the complete diffusion case where $D_{\mathrm{CO}_{2}} \neq 0$ and $D_{\mathrm{CH}_{4}} \neq 0$. We set

$$
\begin{aligned}
& \overline{\bar{D}}_{5}=\left\{D_{X}=0.005, \quad D_{S}=0.007, \quad D_{B}=0.009\right\} \\
& \overline{\bar{D}}_{5}^{c}=\left\{D_{X}=0.005, \quad D_{S}=0.007, \quad D_{B}=0.009, \quad D_{C O_{2}}=0.009, \quad D_{C H_{4}}=0.009\right\}
\end{aligned}
$$

In Figures (9) and (10), we notice that the concentration of $\mathrm{CH}_{4}$ is more smooth (regularizing effect) but its average value do not increases. Besides, the CPU time is smaller 


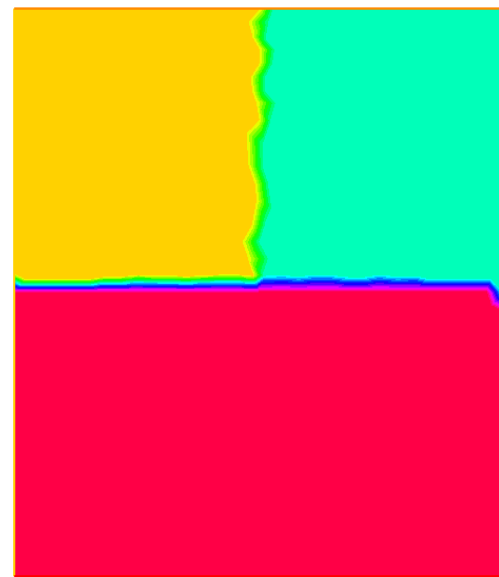

(a)- $\overline{\bar{D}}=\overline{\bar{D}}_{0}$ (no diffusion term)

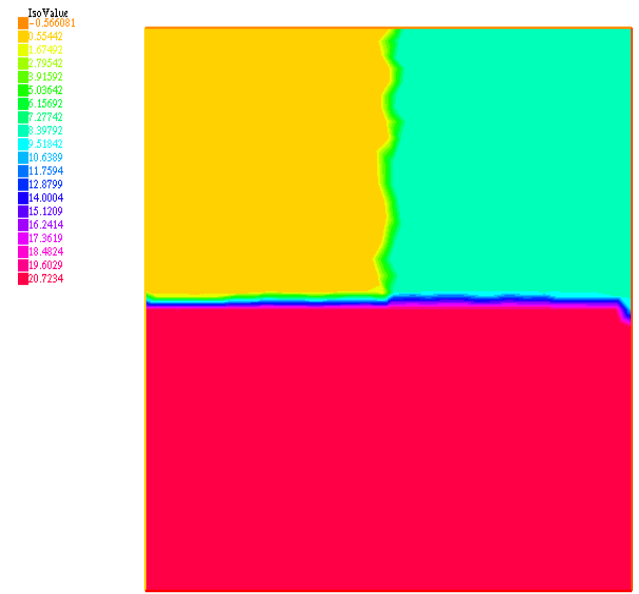

(b) $-\overline{\bar{D}}=\overline{\bar{D}}_{2}$

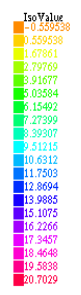

Figure 7: The production of methane after 2 days using the Haldane law without the diffusion in (a) and with the diffusion $\overline{\bar{D}}_{2}$ in (b).

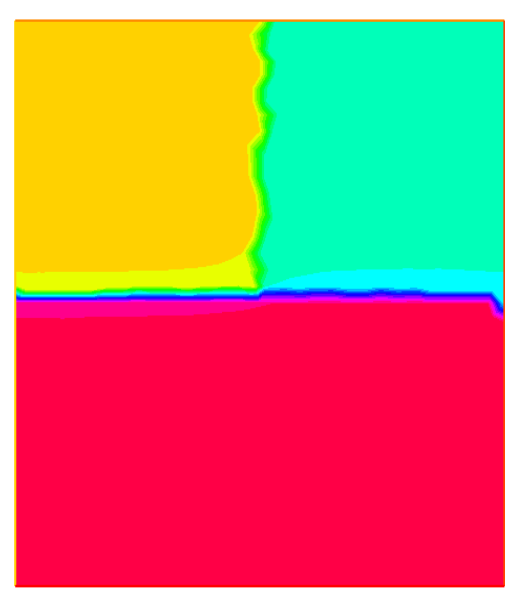

(c)- $\overline{\bar{D}}=\overline{\bar{D}}_{3}$

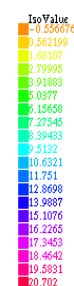

(d) $-\overline{\bar{D}}=\overline{\bar{D}}_{4}$
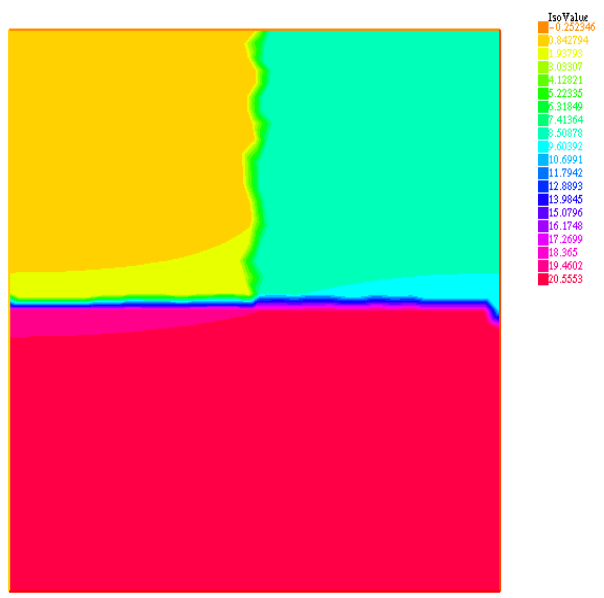

Figure 8: The production of methane after 2 days using Haldane law with the diffusion $\overline{\bar{D}}_{3}$ in (c) and $\overline{\bar{D}}_{4}$ in (d).

with the partial diffusion, (7.605s in 2D and 71s in 3D), than with the complete one (8.7s in $2 \mathrm{D}$, and $103 \mathrm{~s}$ in $3 \mathrm{D}$ ). Therefore the partial diffusion seems more suitable choice in 3D. 


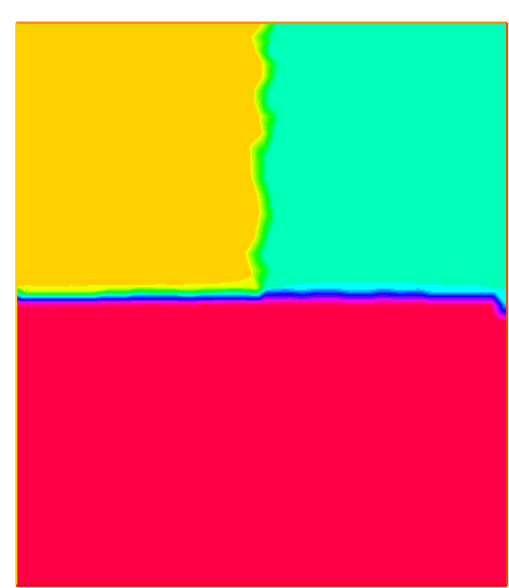

$\overline{\bar{D}}_{5}$

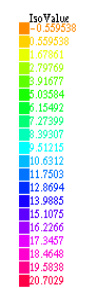

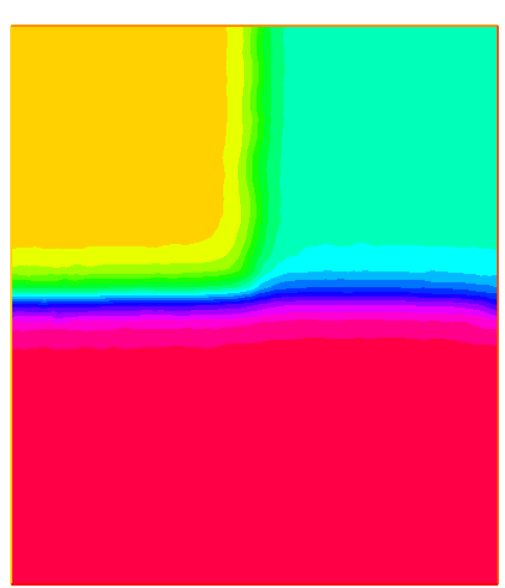

$\overline{\bar{D}}_{5}^{c}$

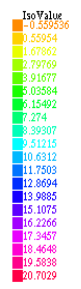

Figure 9: The production of methane after 2 days using Haldane law with $\overline{\bar{D}}_{5}$ and $\overline{\bar{D}}_{5}^{c}$ in 2D.

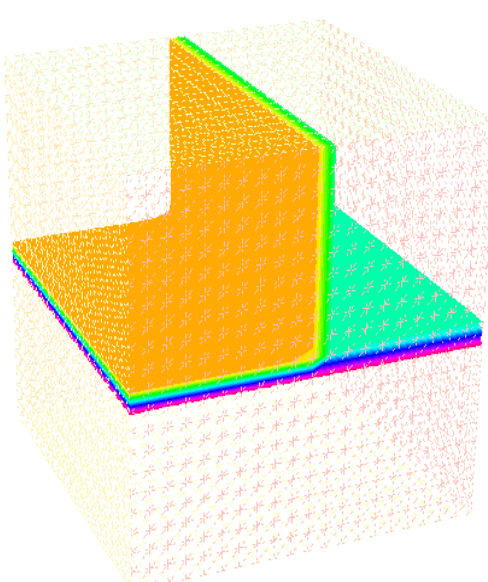

$\overline{\bar{D}}_{5}$

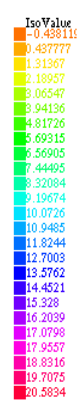

$\overline{\bar{D}}_{5}^{c}$

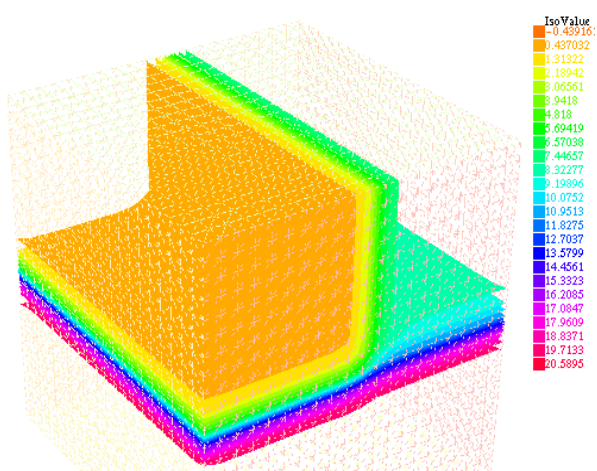

Figure 10: The production of methane after 2 days using Haldane law with $\overline{\bar{D}}_{5}$ and $\overline{\bar{D}}_{5}^{c}$ in 3D.

\subsection{Solution of the full problem (S1)-(S2)}

We turn now to the full system (S1)-(S2) and we take Haldane law and $\overline{\bar{D}}_{3}$ as a diffusion term. We set $B_{0}$

$$
B_{0}(x, y)=\left\{\begin{array}{lll}
1 & \text { in } \quad\{0 x \leq 0.5,0.5 \leq y \leq 1 \text { and } 0.5 \leq z \leq 1\} \\
2 & \text { in }\{0.5 \leq x \leq 1,0.5 \leq y \leq 1 \text { and } 0.5 \leq z \leq 1\} \\
3 & \text { in }\{0 \leq x \leq 1 \mathrm{n} 0<y<0.5 \text { and } 0 \leq z<0.5\}
\end{array}\right.
$$

Figures (12), (13) and (14) show respectively, the velocity field of the Darcy flow, the pressure head, and the water content (via the function $\theta(p)$ ). We notice that after 2 days 
there is a stagnation zone of the leachate (i.e the liquid phase) in the bottom indicating that the medium is saturated therefore there is no biogas production there (we recall that the humidity rate $\omega$ lies between $\omega_{1}$ and $\omega_{2}$ ). It follows that injecting water in the landfill will improve the biogas production which should be addressed in a more precise way with an optimal control approach.

This also suggests to investigate more deeply the role of the humidity rate $\omega$ (see Figure (2)) in the modelling.

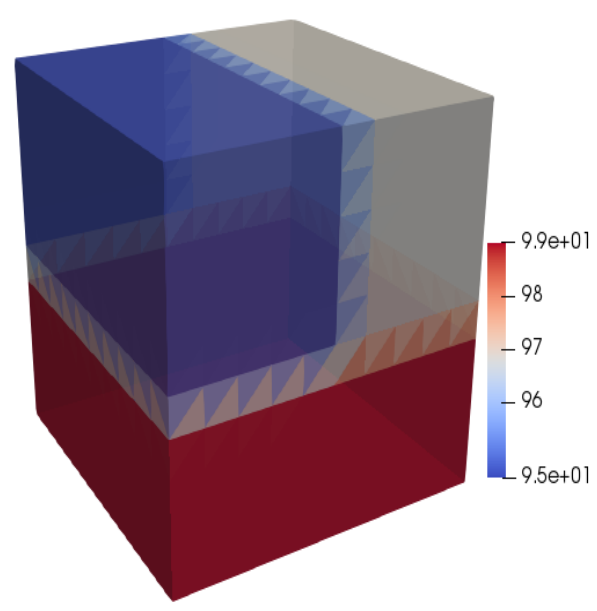

(a) 1 day

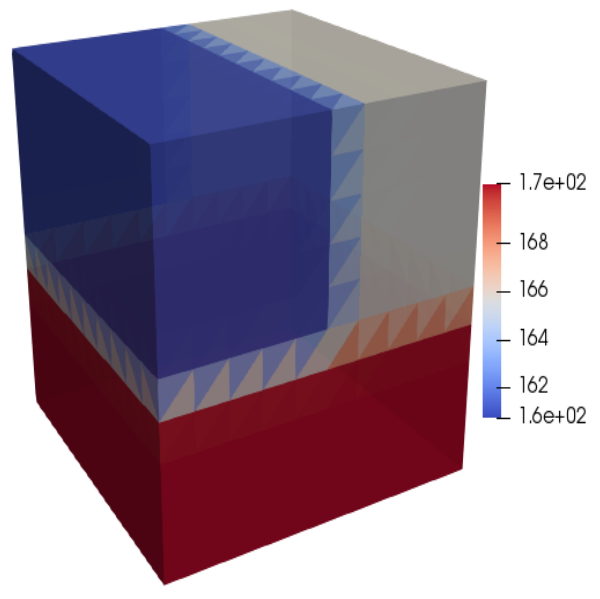

(b) 2 days

Figure 11: Evolution of biogas production with $\overline{\bar{D}}_{3}$ and Haldane law.

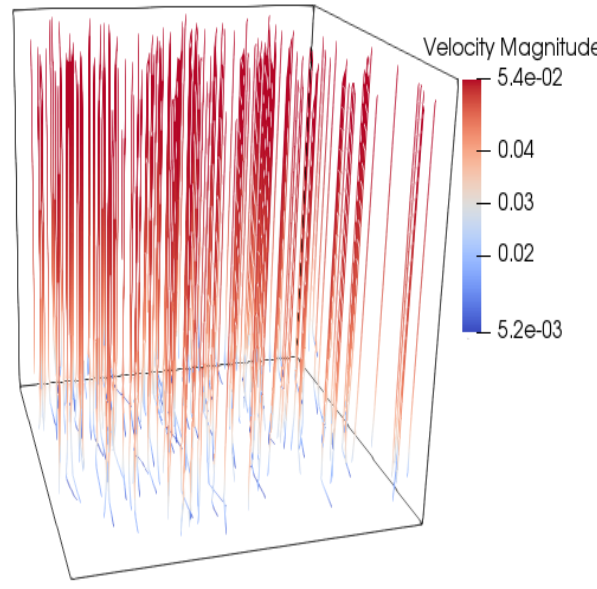

(a) 1 day

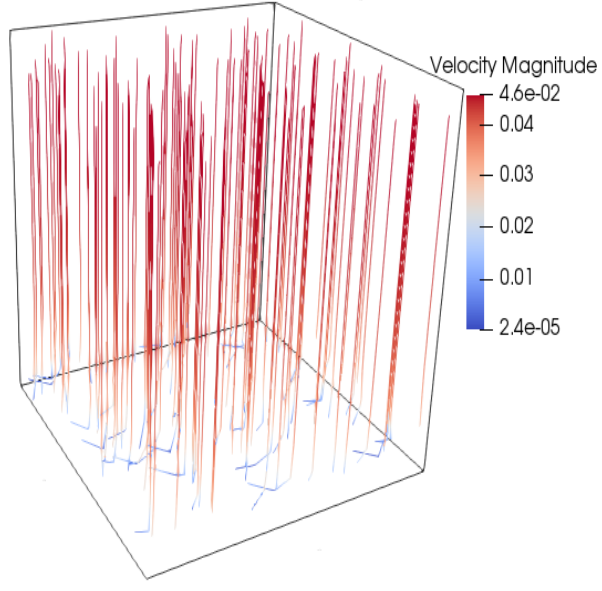

(b) 2 days

Figure 12: The velocity of leachate (liquid phase) with $\overline{\bar{D}}_{3}$ and Haldane law. 


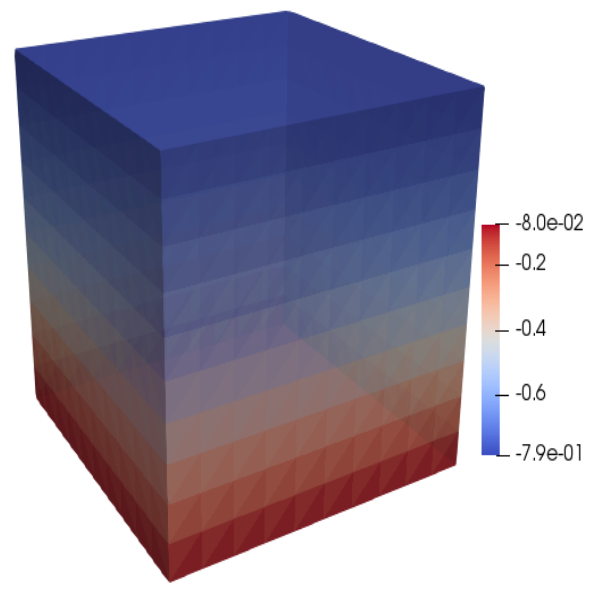

(a) 1 day

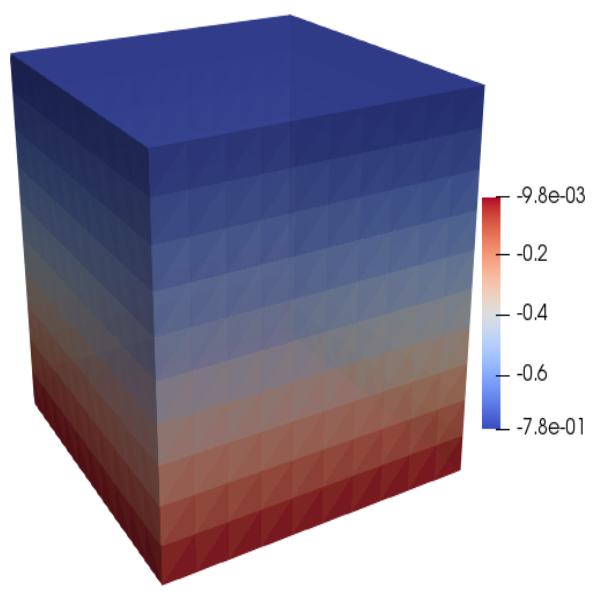

(b) 2 days

Figure 13: Evolution of the pressure head $p$ with $\overline{\bar{D}}_{3}$ and Haldane law.

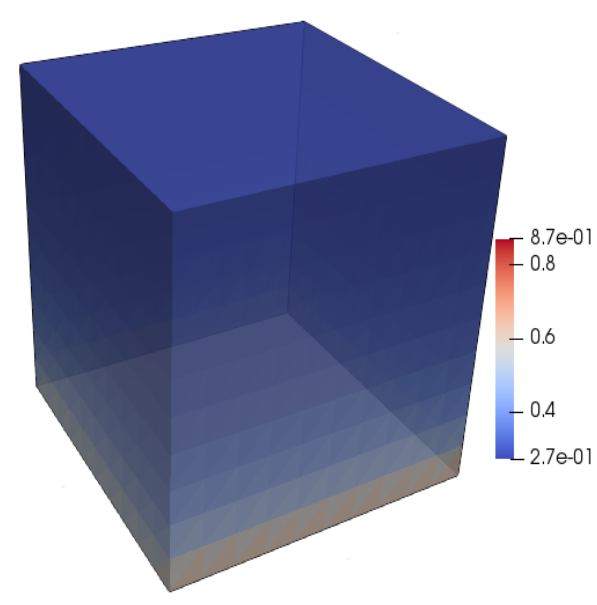

(a) 1 day

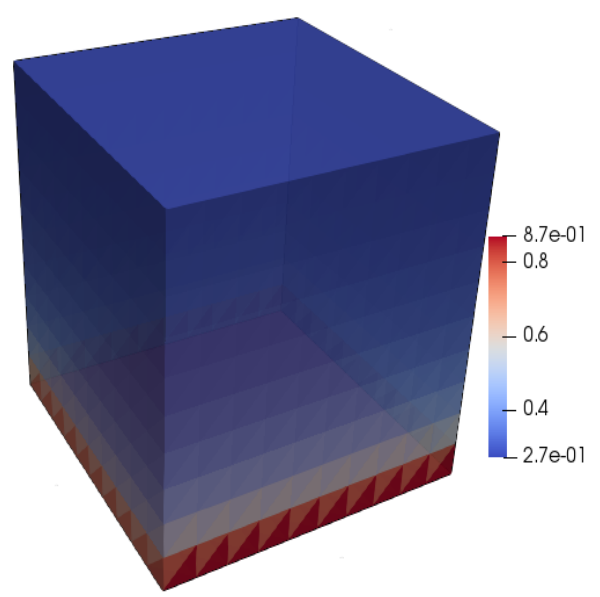

(b) 2 days

Figure 14: The water content evolution with $\overline{\bar{D}}_{3}$ and Haldane law.

\section{Conclusion}

In this article we have considered a coupled system of ODE-PDEs representing the bacterial activity in a landfill taken as an unsaturated and inhomogeneous porous medium. We have introduced new diffusion terms in the ODEs system in order to take into account the spatial evolution of the bacterial dynamic. We analyzed the new model of reactiondiffusion coupled with Darcy flow system and we carried out the analysis of the discrete systems with finite element method. We obtained well-posedness and a priori error estimates for the discrete problems. Finally, we have performed several numerical 2D and 3D simulations which are in agreement with the theory and the literature. In particular, we 
obtain that the introduction of the additional diffusion in the bacterial dynamic improves the existing models and enforces the accuracy of the coupling with the porous medium flow. The discrete problems with a BDF2 scheme and a mixed finite element formulation with $R T_{0} / P_{0}$ constitute a highly efficient numerical method to solve such a complex problem. In a forthcoming paper we extend this approach to a two phase flow where the gaseous phase is included in the model. Moreover, for more realistic models in waste management and renewable energy production, the transport of the biogas should be considered and the approach followed in this article seems to be the right way to do so.

\section{Acknowledgments}

The authors are gratefully for the French-Moroccan exchange program "PHC TOUBKAL $17 / 47^{\prime \prime}$.

\section{References}

[1] Agostini, F., SundBerg, C., NAVIA, R. (2012) ,Is biodegradable waste a porous environment? A review. Waste Management, Research, 30(10), 1001-1015.

[2] Alt, H. W., Luckhaus, S. (1983).,Quasilinear elliptic-parabolic differential equations. Mathematische Zeitschrift, 183(3), 311-341.

[3] ArAn, C. (2001), Modélisation des Ecoulements de Fluides et des Transferts de Chaleur au Sein des Déchets Ménagers. Application à la Réinjection de Lixiviat dans un Centre de Stockage. (Doctoral dissertation)

[4] J. Arzate, M. Kirstein, F. Ertem, E. Kielhorn, H. Malule, P. Neubauer, M. CruzBOURNAZOU AND S. JUNNE (2017),Anaerobic digestion model (AM2) for the description of biogas processes at dynamic feedstock loading rates. Chemie Ingenieur Technik, 89(5), 686-695.

[5] Belhachmi, Z., BuCUR, D., SAC-EPEE, J. M. (2006) ,Finite element approximation of the Neumann eigenvalue problem in domains with multiple cracks. IMA journal of numerical analysis, 26(4), 790-810.

[6] BenYAHia, B., SARI, T., Cherki, B., HARMAND, J. (2012), Bifurcation and stability analysis of a two step model for monitoring anaerobic digestion processes. Journal of Process Control, 22(6), 1008-1019.

[7] Bernard, O., Hadj-Sadok, Z., Dochain, D., Genovesi, A., Steyer, J. P. (2001), Dynamical model development and parameter identification for an anaerobic wastewater treatment process. Biotechnology and bioengineering, 75(4), 424-438.

[8] Boffi, D., Brezzi, F., Fortin, M. (2013), Mixed finite element methods and applications. (Vol. 44, pp. xiv-685). Heidelberg: Springer.

[9] Bothe, D., Fischer, A., Pierre, M., Rolland, G. (2017), Global wellposedness for a class of reaction-advection-anisotropic-diffusion systems. Journal of Evolution Equations, 17(1), 101130.

[10] Brezzi, F., AND Fortin, M. (2012), Mixed and hybrid finite element methods. (Vol. 15). Springer Science and Business Media.

[11] Brooks, R.H., CoreY., A.T. (1964), Hydraulic Properties of Porous Media, Colorado State Univ. Hydrology Paper No. 3., 27 pp.

[12] CAMPBELL, G. S. (1974),A simple method for determining unsaturated conductivity from moisture retention data. Soil science, 117(6), 311-314. 
[13] CARrayrou, J., MosÉ, R., BeHRA, P. (2004), Operator-splitting procedures for reactive transport and comparison of mass balance errors. Journal of Contaminant Hydrology, 68(3-4), 239-268.

[14] Celia, M. A., Kindred, J. S., Herrera, I. (1989), Contaminant transport and biodegradation: 1. A numerical model for reactive transport in porous media. Water Resources Research, 25(6), 1141-1148.

[15] Chenu, D. (2007), Modélisation des transferts réactifs de masse et de chaleur dans les installations de stockage de déchets ménagers: application aux installations de type bioréacteur. (Doctoral dissertation, Institut National Polytechnique de Toulouse).

[16] DIDI, I., DIB, H., CHERKI, B. (2015), A Luenberger-type observer for the AM2 model. Journal of Process Control, 32, 117-126.

[17] Dollé, G., Duran, O., Feyeux, N., FrÉnod, E., Giacomini, M., And Prud’Homme, C. (2016), Mathematical modeling and numerical simulation of a bioreactor landfill using Feel++. ESAIM: Proceedings and Surveys, 55, 83-110.

[18] Fekih-Salem, R., Harmand, J., Lobry, C., RAPAport, A., SARi, T. (2013), Extensions of the chemostat model with flocculation. Journal of Mathematical Analysis and Applications, 397(1), 292-306.

[19] Fortin, M., Mghazli, Z. (1992), Analyse d'un élément mixte pour le problème de Stokes. Numerische Mathematik, 62(1), 161-188.

[20] Gallo, C. AND Manzini, G. (2001), A fully coupled numerical model for two-phase flow with contaminant transport and biodegradation kinetics. Communications in Numerical Methods in Engineering, 17(5), 325-336.

[21] Ghogomu, F. N. (2002), Simulation du transport réactif dans un milieu poreux discrètement fracturé.

[22] GHolamifard, S. (2009) , Modélisation des écoulements diphasiques bioactifs dans les installations de stockage de déchets. (Doctoral dissertation, Université Paris-Est).

[23] Gil Diaz, J. L., Szantò Narea, M., Sanchez Alciturri, J. M., Arias ibarra, A., Tejero Monzòn, I., Palma Gonzalez, J., FAntelli Lamia, M. (1995), Estimating material losses in sanitary landfills through biological degradation. In Sardinia 95, Proceedings of the Fifth International Landfill Symposium (pp. 203-208).

[24] Girault, V., RaViart, P. A. (2012),Finite element methods for Navier-Stokes equations: theory and algorithms (Vol. 5). Springer Science, Business Media.

[25] S. HASSAM, E. FICARA, A. LEVA AND J. HARMAND, A generic and systematic procedure to derive a simplified model from the anaerobic digestion model, No. 1 (ADM1), Biochemical Engineering Journal, 99 (2015), 193-203.

[26] F. Hecht, O. Pironneau, J. Morice, FreefEM++, v3.20, third edition, 2013, Www.freefem.org.

[27] Hmissi, M., Harmand, J., AlCARAZ-Gonzalez, V., Shayeb, H. (2017), Evaluation of alkalinity spatial distribution in an up-flow fixed bed anaerobic digester. Water Science and Technology, 77(4), 948-959.

[28] Kindlein, J., Dinkler, D., Ahrens, H. (2006) ,Numerical modelling of multiphase flow and transport processes in landfills. Waste Management and Research, 24(4), 376-387.

[29] LANini, S. (1998), Analyse et modélisation des transferts de masse et de chaleur au sein des décharges d'ordures ménagères. (Doctoral dissertation, Institut National Polytechnique de Toulouse).

[30] Nguyen-Ngoc, D., Leye, B., Monga, O., Garnier, P., And NunAn, N. (2013),Modeling microbial decomposition in real $3 D$ soil structures using partial differential equations. International Journal of geosciences, 4(10), 15.

[31] S. Ouchtout, Z. Mghazl, J. Harmand, A. Rapaport, Z. Belhachmi,Analysis of an anaerobic 
digestion model in landfill with mortality term. Communications on Pure and Applied Analysis, 2020, 19 (4) : 2333-2346. doi: 10.3934/cpaa.2020101

[32] Pinder, G. F., GRAY, W. G. (2008), Essentials of multiphase flow and transport in porous media. John Wiley and Sons.

[33] A. RAPAPORT, T. Nidelet, S. El AidA AND J. HARMAnd, About biomass overyielding of mixed cultures in batch processes, Prepint hal, (2019).

[34] A. RAPAPORT, T. Nidelet AND J. HARMAnd, About biomass overyielding of mixed cultures in batch processes, in 8th IFAC Conference on Foundations of Systems Biology in Engineering (FOSBE), Valencia, Spain, 15-18 Oct., (2019).

[35] Richards, L. A. (1931), Capillary conduction of liquids through porous mediums. Physics, 1(5), 318-333.

[36] Rouez, M. (2008), Dégradation anaérobie de déchets solides: Caractérisation, facteurs d’influence et modélisations. Laboratoire de Génie Civil et d'Ingénierie Environnementale. Lyon, Institut National des Sciences Appliquées Docteur, 259.

[37] SHI, J., Wu, Y., Zou, X. (2019), Coexistence of Competing Species for Intermediate Dispersal Rates in a Reaction Diffusion Chemostat Model. Journal of Dynamics and Differential Equations, $1-28$.

[38] Tinet, A. J. (2011), Contribution à l'étude des transferts de fluides dans les installations de stockage des déchets non dangereux. (Doctoral dissertation, Université Grenoble Alpes).

[39] VAN GENUCHTEN, M. T. (1980),A closed-form equation for predicting the hydraulic conductivity of unsaturated soils 1. Soil science society of America journal, 44(5), 892-898.

[40] VAnrolleghem, P. A., Dochain, D. (2001),Dynamical Modelling and Estimation in Wastewater Treatment Processes. IWA Publishing. 